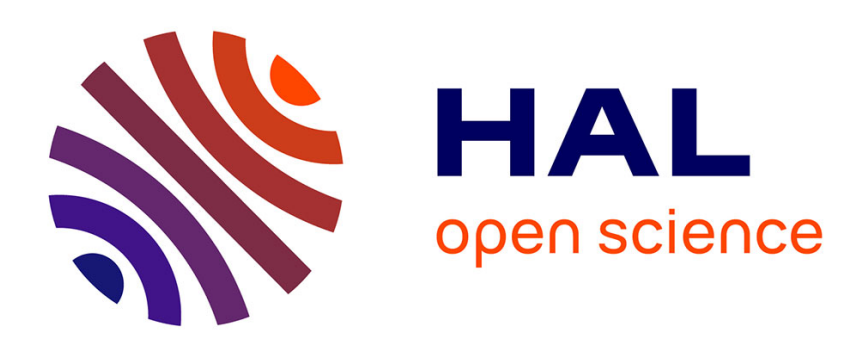

\title{
On cross-diffusion systems for two populations subject to a common congestion effect
}

\author{
Maxime Laborde
}

\section{To cite this version:}

Maxime Laborde. On cross-diffusion systems for two populations subject to a common congestion effect. 2019. hal-01593657v2

\section{HAL Id: hal-01593657 \\ https://hal.science/hal-01593657v2}

Preprint submitted on 1 Mar 2019

HAL is a multi-disciplinary open access archive for the deposit and dissemination of scientific research documents, whether they are published or not. The documents may come from teaching and research institutions in France or abroad, or from public or private research centers.
L'archive ouverte pluridisciplinaire HAL, est destinée au dépôt et à la diffusion de documents scientifiques de niveau recherche, publiés ou non, émanant des établissements d'enseignement et de recherche français ou étrangers, des laboratoires publics ou privés. 


\title{
On cross-diffusion systems for two populations subject to a common congestion effect
}

\author{
Maxime Laborde*
}

\begin{abstract}
In this paper, we investigate the existence of solution for systems of Fokker-Planck equations coupled through a common nonlinear congestion. Two different kinds of congestion are considered: a porous media congestion or soft congestion and the hard congestion given by the constraint $\rho_{1}+\rho_{2} \leqslant 1$. We show that these systems can be seen as gradient flows in a Wasserstein product space and then we obtain a constructive method to prove the existence of solutions. Therefore it is natural to apply it for numerical purposes and some numerical simulations are included.
\end{abstract}

Keywords: Wasserstein gradient flows, Jordan-Kinderlehrer-Otto scheme, crowd motion, nonlinear cross-diffusion systems.

MS Classification: 35K40, 49J40, 49J45.

\section{Introduction}

The modelling of crowd behaviour has become a very active field of applied mathematics in recent years. These models permit to understand many phenomena such as cell migration, tumor growth, etc. Several models already exist to tackle this problem. The first one, microscopic, consists in seeing a population as a high number of individuals which satisfy ODEs, see for instance [36] and the second is macroscopic and consists in describing a population by a density $\rho$ satisfying a PDE, where $\rho(t, x)$ represents the density of individuals in $x$ at time $t$. In the latter framework, different methods to handle the congestion effect have been proposed. The first one consists in saying that the motion has to be slower when the density is very high, see for example [14, 13, 12 for a different approach with applications to crowd dynamics. Another way of modelling the congestion effect is to use a threshold: the density evolves as we would expect until it touches a maximal level and then the motion has to be adapted in these regions (to not increase the density there), see for example [33] for crowd motion model and [34] for application to dendritic growth. For a comparison between microscopic and macroscopic models, we refer to [35]. In [38, Mészáros and Santambrogio proposed a model for hard congestion where individuals are subject to a Brownian diffusion. This corresponds to modified a Fokker-Planck equation with an $L^{\infty}$ constraint on the density.

Since in macroscopic models, we have mass conservation, the theory of optimal transportation is a very natural tool to attack them. In [33], the authors investigated a model of room evacuation. They showed that if the desired velocity field of the individuals is given by a gradient, say $V=\nabla D$, where $D$ is the distance to a given target, then the problem has a gradient flow structure in the Wasserstein space and the velocity field has to be adapted by a pressure field to handle congestion effect. More recently in [38, a splitting scheme has been introduced to handle velocity fields which are not necessarily gradient field. The scheme consists in combining steps where the density follows the unconstrained Fokker-Planck equation and Wasserstein projections onto the set of densities which cannot exceed 1.

*Department of Mathematics and Statistics, McGill University, Montreal, CANADA (maxime.laborde@mcgill.ca) 
A natural variant of the model of [38], consists in considering two (or more) populations, each of whom is subject to an advection term coming from different potential gradients but coupled through the constraint that the total density cannot exceed a given threshold, say 1, and then subject to a common pressure field. Note that variant problems with total density equal to 1 are treated in [15, 4, 5, 9] and for more general cross-diffusion systems, we refer, for instance, to [31, 18, 24, 25, 27]. For a linear diffusion (corresponding to a Brownian noise on each species), the two-species crowd dynamic is expressed by the PDEs

$$
\left\{\begin{array}{l}
\partial_{t} \rho_{1}-\Delta \rho_{1}-\operatorname{div}\left(\rho_{1}\left(\nabla V_{1}+\nabla p\right)\right)=0 \\
\partial_{t} \rho_{2}-\Delta \rho_{2}-\operatorname{div}\left(\rho_{2}\left(\nabla V_{2}+\nabla p\right)\right)=0 \\
p \geqslant 0, \rho_{1}+\rho_{2} \leqslant 1, p\left(1-\rho_{1}-\rho_{2}\right)=0 \\
\rho_{1}(0, \cdot)=\rho_{1,0}, \rho_{2}(0, \cdot)=\rho_{2,0}
\end{array}\right.
$$

on $\Omega$ a convex compact subset of $\mathbb{R}^{n}$ with smooth boundary such that

$$
|\Omega|>2
$$

The assumption 1.2 is made to ensure that the subset

$$
\mathcal{K}:=\left\{\left(\rho_{1}, \rho_{2}\right) \in \mathcal{P}^{\mathrm{ac}}(\Omega)^{2}: \rho_{1}+\rho_{2} \leqslant 1 \text { a.e. }\right\}
$$

is neither empty nor trivial. We put no-flux boundary conditions to preserve the mass in $\Omega$,

$$
\left(\nabla \rho_{1}+\rho_{1}\left(\nabla V_{1}+\nabla p\right)\right) \cdot \nu=0 \text { and }\left(\nabla \rho_{2}+\rho_{2}\left(\nabla V_{2}+\nabla p\right)\right) \cdot \nu=0 \text { a.e. on } \mathbb{R}^{+} \times \partial \Omega,
$$

where $\nu$ is the outward unit normal to $\partial \Omega$.

In this paper, we show that this system is the gradient flow for the Wasserstein product distance of the energy

$$
\mathcal{E}_{\infty}\left(\rho_{1}, \rho_{2}\right):= \begin{cases}\sum_{i=1}^{2} \int_{\Omega}\left(\rho_{i} \log \left(\rho_{i}\right)+V_{i} \rho_{i}\right)+\int_{\Omega} \chi_{[0,1]}\left(\rho_{1}(x)+\rho_{2}(x)\right) \mathrm{d} x & \text { if } \rho_{i} \log \left(\rho_{i}\right) \in L^{1}(\Omega), \\ +\infty, & \text { otherwise },\end{cases}
$$

where $\chi_{[0,1]}$ is the indicator function of $[0,1]$,

$$
\chi_{[0,1]}(z):= \begin{cases}0 & \text { if } z \in[0,1] \\ +\infty & \text { otherwise. }\end{cases}
$$

In addition, for a different energy of the form

$$
\begin{aligned}
& \mathcal{E}_{m}\left(\rho_{1}, \rho_{2}\right):= \\
& \begin{cases}\sum_{i=1}^{2} \int_{\Omega}\left(\rho_{i} \log \left(\rho_{i}\right)+V_{i} \rho_{i}\right)+\int_{\Omega} \frac{1}{m-1}\left(\rho_{1}(x)+\rho_{2}(x)\right)^{m} d x & \text { if } \rho_{i} \log \left(\rho_{i}\right),\left(\rho_{1}+\rho_{2}\right)^{m} \in L^{1}(\Omega), \\
+\infty & \text { otherwise },\end{cases}
\end{aligned}
$$

for $m>1$, the gradient flow of $\mathcal{E}_{m}$ leads to the following nonlinear system

$$
\left\{\begin{array}{l}
\partial_{t} \rho_{1}=\Delta \rho_{1}+\operatorname{div}\left(\rho_{1} \nabla\left(V_{1}+\frac{m}{m-1}\left(\rho_{1}+\rho_{2}\right)^{m-1}\right)\right) \\
\partial_{t} \rho_{2}=\Delta \rho_{2}+\operatorname{div}\left(\rho_{2} \nabla\left(V_{2}+\frac{m}{m-1}\left(\rho_{1}+\rho_{2}\right)^{m-1}\right)\right) \\
\rho_{1}(0, \cdot)=\rho_{1,0}, \rho_{2}(0, \cdot)=\rho_{2,0}
\end{array}\right.
$$

with no flux boundary conditions. Then for a given small time step $h>0$, the JKO scheme for this energy reads,

$$
\left(\rho_{1}^{k+1}, \rho_{2}^{k+1}\right)=\underset{\left(\rho_{1}, \rho_{2}\right)}{\operatorname{argmin}}\left\{\sum_{i=1}^{2} \frac{1}{2 h} W_{2}^{2}\left(\rho_{i}, \rho_{i}^{k}\right)+\mathcal{E}_{m}\left(\rho_{1}, \rho_{2}\right)\right\}
$$

which, in the particular case of the linear diffusion crowd motion problem with two species, takes the form 


$$
\left(\rho_{1}^{k+1}, \rho_{2}^{k+1}\right)=\underset{\rho_{1}+\rho_{2} \leqslant 1}{\operatorname{argmin}}\left\{\sum_{i=1}^{2}\left(\frac{1}{2 h} W_{2}^{2}\left(\rho_{i}, \rho_{i}^{k}\right)+\int_{\Omega}\left(\rho_{i} \log \left(\rho_{i}\right)+V_{i} \rho_{i}\right)\right)\right\} .
$$

We want to mention that the results in this paper have been obtained in the authors's $\mathrm{PhD}$ thesis, 28, back in 2016. Note that recently, in [26, Kim and Mészáros studied problems (1.3) and (1.1) without individual diffusions. They prove existence of weak solution in dimension 1 for segregated initial conditions and ordered drifts. In any dimension, they prove existence of very weak solutions. The difficulty is to handle the cross diffusive term which needs to have strong compactness in $\rho_{1}, \rho_{2}$ and $\rho_{1}+\rho_{2}$. Here, this difficulty is overcome by assuming that individuals of each populations are subject to a Brownian diffusion. This allows us to obtain separated estimates on $\rho_{i}$ and $\rho_{1}+\rho_{2}$. In [30, Laurençot and Matioc give a similar result in $\mathbb{R}$ and $m=2$. In this paper, we extend this result on $\Omega \subset \mathbb{R}^{n}$ and with $m \in[1,+\infty]$. Furthermore, taking advantage of the gradient flow structure, we give numerical simulations implemented by the augmented Lagrangian scheme introduced in [6]. We want to point out that uniqueness of systems $(1.1)$ and $(1.3)$ is still an open question due to the lack of geodesic convexity of the common energy and we do not adress this problem in this paper. We refer to [26] for further discussions on this subject.

This paper is organized as follows. In section 2, we introduce our assumptions and we state our main results. In section 3, we prove the existence of a weak solution for system (1.3). The key ingredient is the flow interchange argument (see [32, 20, 29] for example) which gives separated estimates on the gradient of $\rho_{1}+\rho_{2}$ and on the gradient of $\rho_{i}$. Section 4 provides the proof of existence of a weak solution for system (1.1). In this section we use again the flow interchange argument to obtain stronger estimates. In section 5 , we focus on the particular case where $\nabla V_{1}=$ $\nabla V_{2}$. In this case, we are able to show the convergence when $m \rightarrow+\infty$ of a solution to 1.3 to a solution to 1.1 and we prove a $L^{1}$-contraction theorem. In the final section 6 numerical simulations are presented.

\section{$2 \quad$ Preliminaries and main results}

Throughout the paper, $\Omega$ is a smooth convex bounded subset of $\mathbb{R}^{n}$. We start to recall some results from the optimal tranportation theory and then we will state our main results.

\subsection{Wasserstein space}

For a detailed exposition, we refer to reference textbooks [45, 46, 3, 44]. We denote $\mathcal{M}^{+}(\Omega)$ the set of nonnegative finite Radon measures on $\Omega, \mathcal{P}(\Omega)$ the space of probability measures on $\Omega$, and $\mathcal{P}^{\text {ac }}(\Omega)$, the subset of $\mathcal{P}(\Omega)$ of probability measures on $\Omega$ absolutely continuous with respect to the Lebesgue measure.

For all $\rho, \mu \in \mathcal{P}(\Omega)$, we denote $\Pi(\rho, \mu)$, the set of probability measures on $\Omega \times \Omega$ having $\rho$ and $\mu$ as first and second marginals, respectively, and an element of $\Pi(\rho, \mu)$ is called a transport plan between $\rho$ and $\mu$. Then for all $\rho, \mu \in \mathcal{P}(\Omega)$, we denote by $W_{2}(\rho, \mu)$ the Wasserstein distance between $\rho$ and $\mu$, defined as

$$
W_{2}^{2}(\rho, \mu)=\min \left\{\iint_{\Omega \times \Omega}|x-y|^{2} d \gamma(x, y): \gamma \in \Pi(\rho, \mu)\right\} .
$$

Since this optimal transportation problem is a linear problem under linear constraints, it admits a dual formulation given by

$$
W_{2}^{2}(\rho, \mu)=\sup \left\{\int_{\Omega} \varphi(x) d \rho(x)+\int_{\Omega} \psi(y) d \mu(y): \varphi, \psi \in \mathcal{C}(\Omega) \text { s.t. } \varphi(x)+\psi(y) \leqslant|x-y|^{2}\right\} .
$$

Optimal solutions to the dual problem are called Kantorovich potentials between $\rho$ and $\mu$. If $\rho \in \mathcal{P}^{\text {ac }}(\Omega)$, a well-known result proved by Brenier, [7], states that the optimal transport plan, $\gamma$, is unique and is induced by an optimal transport map, $T$, i.e $\gamma$ is of the form $(I d \times T)_{\#} \rho$, where $T_{\#} \rho=\mu$ and $T$ is the gradient of a convex function. Moreover, the optimal transport map is given 
by $T=I d-\nabla \varphi$ where $(\varphi, \psi)$ is a pair of Kantorovich potentials between $\rho$ and $\mu$.

It is well known that $\mathcal{P}(\Omega)$ endowed with the Wasserstein distance defines a metric space and since $\Omega$ is compact, $W_{2}$ metrizes the narrow convergence of probability measures.

\subsection{Assumptions and main results}

For $i \in\{1,2\}$, we define $\mathcal{V}_{i}: \mathcal{P}(\Omega) \rightarrow \mathbb{R}$ the potential energy associated to $V_{i} \in W^{1, \infty}(\Omega)$ as

$$
\mathcal{V}_{i}(\rho):=\int_{\Omega} V_{i}(x) d \rho(x)
$$

We introduce the Entropy $\mathcal{H}$ defined, for all probabilty measures $\rho$, as

$$
\mathcal{H}(\rho):=\left\{\begin{array}{ll}
\int_{\Omega} H(\rho(x)) d x & \text { if } \rho \ll \mathcal{L}_{\mid \Omega}, \\
+\infty & \text { otherwise, }
\end{array}, \quad H(z):=z \log (z) \text { for all } z \in \mathbb{R}^{+} .\right.
$$

Finally, for $m \in[1,+\infty)$, we define $\mathcal{F}_{m}: \mathcal{M}^{+}(\Omega) \rightarrow \mathbb{R} \cup\{+\infty\}$ as

$\mathcal{F}_{m}(\rho):=\left\{\begin{array}{ll}\int_{\Omega} F_{m}(\rho(x)) d x & \text { if } \rho \ll \mathcal{L}_{\mid \Omega}, \\ +\infty & \text { otherwise, }\end{array}, \quad F_{m}(z):=\left\{\begin{array}{cl}z \log z & \text { if } m=1, \\ \frac{z^{m}}{m-1} & \text { if } m>1 .\end{array}\right.\right.$ for all $z \in \mathbb{R}^{+},(2.1)$

and, for $m=+\infty, \mathcal{F}_{\infty}: \mathcal{M}^{+}(\Omega) \rightarrow \mathbb{R} \cup\{+\infty\}$ is defined by

$$
\mathcal{F}_{\infty}(\rho):= \begin{cases}0 & \text { if }\|\rho\|_{\infty} \leqslant 1 \\ +\infty & \text { otherwise }\end{cases}
$$

Definition 2.1 (Weak solution).

- We say that $\left(\rho_{1}, \rho_{2}\right):[0,+\infty) \rightarrow \mathcal{P}^{\mathrm{ac}}(\Omega)^{2}$ is a weak solution to 1.3 if for all $i \in\{1,2\}$ and for all $T<+\infty, \rho_{i} \in \mathcal{C}^{0,1 / 2}\left([0, T], \mathcal{P}^{\mathrm{ac}}(\Omega)\right) \cap L^{2-1 / m}\left((0, T), W^{1,2-1} m(\Omega)\right) \cap L^{2 m-1}((0, T) \times$ $\Omega), \rho_{i} \nabla F_{m}^{\prime}\left(\rho_{1}+\rho_{2}\right) \in L^{2-1 / m}((0, T) \times \Omega)$ and for all $\phi \in \mathcal{C}_{c}^{\infty}\left([0,+\infty) \times \mathbb{R}^{n}\right)$,

$$
\int_{0}^{+\infty} \int_{\Omega}\left[\rho_{i} \partial_{t} \phi-\left(\rho_{i} \nabla V_{i}+\rho_{i} \nabla F_{m}^{\prime}\left(\rho_{1}+\rho_{2}\right)+\nabla \rho_{i}\right) \cdot \nabla \phi\right] d x d t=-\int_{\Omega} \phi(0, x) \rho_{i, 0}(x) d x
$$

- We say that $\left(\rho_{1}, \rho_{2}, p\right):[0,+\infty) \rightarrow \mathcal{P}^{\mathrm{ac}}(\Omega)^{2} \times H^{1}(\Omega)$ is a weak solution to 1.1 if for all $i \in\{1,2\}$ and for all $T<+\infty, \rho_{i} \in \mathcal{C}^{0,1 / 2}\left([0, T], \mathcal{P}^{\mathrm{ac}}(\Omega)\right) \cap L^{2}\left((0, T), H^{1}(\Omega)\right), p \in$ $L^{2}\left((0, T), H^{1}(\Omega)\right)$ with $p \geqslant 0, \rho_{1}+\rho_{2} \leqslant 1$ and $p\left(1-\rho_{1}-\rho_{2}\right)=0$ a.e. in $[0, T] \times \Omega$. In addition, for all $\phi \in \mathcal{C}_{c}^{\infty}\left([0,+\infty) \times \mathbb{R}^{n}\right)$,

$$
\int_{0}^{+\infty} \int_{\Omega}\left[\rho_{i} \partial_{t} \phi-\left(\rho_{i} \nabla V_{i}+\rho_{i} \nabla p+\nabla \rho_{i}\right) \cdot \nabla \phi\right] d x d t=-\int_{\Omega} \phi(0, x) \rho_{i, 0}(x) d x .
$$

The main results of this paper are

Theorem 2.2. Assume that $\rho_{1,0}, \rho_{2,0} \in \mathcal{P}^{\mathrm{ac}}(\Omega)$ satisfy

$$
\mathcal{H}\left(\rho_{1,0}\right)+\mathcal{H}\left(\rho_{2,0}\right)+\mathcal{F}_{m}\left(\rho_{1,0}+\rho_{2,0}\right)<+\infty,
$$

then (1.3) admits at least one weak solution.

and

Theorem 2.3. Assume that $\Omega$ satisfies 1.2 . If $\left(\rho_{1,0}, \rho_{2,0}\right) \in \mathcal{K}$ satisfies

$$
\mathcal{H}\left(\rho_{1,0}\right)+\mathcal{H}\left(\rho_{2,0}\right)<+\infty
$$

then there exists at least one weak solution to (1.1. 
Remark 2.4 (Remarks on possible extensions:).

- These models can be generalized to more than two species. Moreover, instead of assuming that individuals of different populations take the same space, we can generalize to densities evolving under the constraints on $\alpha_{1} \rho_{1}+\alpha_{2} \rho_{2}$, for $\alpha_{1}, \alpha_{2}>0$. Then system (1.3) becomes

$$
\partial_{t} \rho_{i}=\operatorname{div}\left(\rho_{i} \nabla V_{i}\right)+\Delta \rho_{i}+\alpha_{i} \operatorname{div}\left(\rho_{i} \nabla F_{m}^{\prime}\left(\alpha_{1} \rho_{1}+\alpha_{2} \rho_{2}\right)\right), i=1,2 .
$$

and system with hard congestion becomes

$$
\left\{\begin{array}{l}
\partial_{t} \rho_{1}-\Delta \rho_{1}-\operatorname{div}\left(\rho_{1}\left(\nabla V_{1}+\nabla p\right)\right)=0 \\
\partial_{t} \rho_{2}-\Delta \rho_{2}-\operatorname{div}\left(\rho_{2}\left(\nabla V_{2}+\nabla p\right)\right)=0 \\
p \geqslant 0, \alpha_{1} \rho_{1}+\alpha_{2} \rho_{2} \leqslant 1, p\left(1-\alpha_{1} \rho_{1}-\alpha_{2} \rho_{2}\right)=0
\end{array}\right.
$$

- These results can be generalized to more general velocities. Indeed, using the semi-implicit scheme introduced by DiFrancesco and Fagioli in [19] and developped in [29] or the splitting method introduced in [10], we can treat vector fields depending on the densities and which come not necessarily from a potential. These extensions allow to treat nonlocal interactions between different species, of the form $V_{i}\left[\rho_{1}, \rho_{2}\right]=K_{i, 1} * \rho_{1}+K_{i, 2} * \rho_{2}$ where $K_{i, j} \in W^{1, \infty}$, which are subject to a common congestion effect .

- To simplify the exposition, during the whole paper, we deal with linear self-diffusion terms but it is possible to extend Theorems 2.2 and 2.3 to nonlinear self-diffusions. In particular, we can deal with porous medium diffusion of the form $\Delta \rho_{i}^{q_{i}}$. This can be done replacing the Entropy $\mathcal{H}\left(\rho_{i}\right)$ by the functional $\mathcal{F}_{q_{i}}\left(\rho_{i}\right)$. In the analysis, the individual estimates found in Proposition 3.6 and in Proposition 4.3 become $L^{2}\left((0, T), H^{1}(\Omega)\right)$ estimates on $\rho_{1, h}^{q_{1} / 2}$ and $\rho_{2, h}^{q_{2} / 2}$ (see for example [29]) without modifying the joint estimate. In addition, discret solutions are not globally supported anymore, i.e. Lemma 3.9 and Lemma 4.4 do not hold, but Proposition 3.10 and Proposition 4.7 can be recovered, see for example [44] for $m<+\infty$ and [26] in the case $m=\infty$.

\section{Coupling through common soft congestion}

In this section, we prove Theorem 2.2 using the implicit JKO scheme, firstly introduced by Jordan, Kinderlherer and Otto in [23. Given a time step $h>0$, we construct by induction two sequences $\rho_{1, h}^{k}$ and $\rho_{2, h}^{k}$ with the following scheme: $\rho_{i, h}^{0}=\rho_{i, 0}$ and for all $k \geqslant 0$,

$$
\left(\rho_{1, h}^{k+1}, \rho_{2, h}^{k+1}\right) \in \underset{\left(\rho_{1}, \rho_{2}\right) \in \mathcal{P}^{\mathrm{ac}}(\Omega)^{2}}{\operatorname{argmin}}\left\{\sum_{i=1}^{2}\left(W_{2}^{2}\left(\rho_{i}, \rho_{i, h}^{k}\right)+2 h\left(\mathcal{H}\left(\rho_{i}\right)+\mathcal{V}_{i}\left(\rho_{i}\right)\right)\right)+2 h \mathcal{F}_{m}\left(\rho_{1}+\rho_{2}\right)\right\} .
$$

These sequences are well-defined by standard compactness and l.s.c argument. Then we define the piecewise constant interpolations $\rho_{i, h}: \mathbb{R}^{+} \rightarrow \mathcal{P}^{\text {ac }}(\Omega)$ by

$$
\rho_{i, h}(t):=\rho_{i, h}^{k+1}, \quad \text { if } t \in(k h,(k+1) h] .
$$

In the first part of this section, we study the convergence of these sequences and then we give the proof of Theorem 2.2 .

\subsection{Estimates and convergences}

We start retrieving classical estimates coming from the JKO scheme, 23], and then, we develop stronger estimates using the flow interchange argument, [32, 20]. First, the minimization scheme gives 
Proposition 3.1. For all $T<+\infty$ and for all $i \in\{1,2\}$, there exists a constant $C<+\infty$ such that for all $k \in \mathbb{N}$ and for all $h$ with $k h \leqslant T$ and let $N=\left\lfloor\frac{T}{h}\right\rfloor$, we have

$$
\begin{gathered}
\mathcal{H}\left(\rho_{i, h}^{k}\right) \leqslant C, \\
\mathcal{F}_{m}\left(\rho_{1, h}^{k}+\rho_{2, h}^{k}\right) \leqslant C, \\
\sum_{k=0}^{N-1} W_{2}^{2}\left(\rho_{i, h}^{k}, \rho_{i, h}^{k+1}\right) \leqslant C h .
\end{gathered}
$$

Proof. These results are obtained easily taking $\rho_{i}=\rho_{i, h}^{k}$ as competitors in [3.1], see [23].

Remark 3.2. Notice that estimate (3.4) does not depend on $m$. This Remark will be useful in section 5 to show that a solution to 1.3 converges to a solution to (1.1).

In the next proposition, stronger estimates are obtained in order to pass to the limit in the nonlinear diffusive term. The main argument to prove this proposition is the flow interchange argument, introduced in [32]. First we recall the definition of a $\kappa$-flow.

Definition 3.3. A semigroup $\mathfrak{S}_{\Psi}: \mathbb{R}^{+} \times \mathcal{P}^{a c}(\Omega) \rightarrow \mathcal{P}^{a c}(\Omega)$ is a $\kappa$-flow for the functional $\Psi$ : $\mathcal{P}^{a c}(\Omega) \rightarrow \mathbb{R} \cup\{+\infty\}$ with respect to $W_{2}$ if, for all $\rho \in \mathcal{P}^{a c}(\Omega)$, the curve $s \mapsto \mathfrak{S}_{\Psi}^{s}[\rho]$ is absolutely continuous on $\mathbb{R}^{+}$and satisfies the evolution variational inequality (EVI)

$$
\left.\frac{1}{2} \frac{d^{+}}{d \sigma}\right|_{\sigma=s} W_{2}^{2}\left(\mathfrak{S}_{\Psi}^{s}[\rho], \tilde{\rho}\right)+\frac{\kappa}{2} W_{2}^{2}\left(\mathfrak{S}_{\Psi}^{s}[\rho], \tilde{\rho}\right) \leqslant \Psi(\tilde{\rho})-\Psi\left(\mathfrak{S}_{\Psi}^{s}[\rho]\right)
$$

for all $s>0$ and for all $\tilde{\rho} \in \mathcal{P}^{a c}(\Omega)$ such that $\Psi(\tilde{\rho})<+\infty$, where

$$
\frac{d^{+}}{d t} f(t):=\limsup _{s \rightarrow 0^{+}} \frac{f(t+s)-f(t)}{s} .
$$

In [3], the authors showed that the fact a functional admits a $\kappa$-flow is equivalent to $\kappa$ displacement convexity.

Proposition 3.4. For all $T>0$, there exists a constant $C_{T}>0$ such that,

$$
\left\|\rho_{1, h}^{1 / 2}\right\|_{L^{2}\left((0, T), H^{1}(\Omega)\right)}^{2}+\left\|\rho_{2, h}^{1 / 2}\right\|_{L^{2}\left((0, T), H^{1}(\Omega)\right)}^{2}+\frac{1}{m}\left\|\left(\rho_{1, h}+\rho_{2, h}\right)^{m / 2}\right\|_{L^{2}\left((0, T), H^{1}(\Omega)\right)}^{2} \leqslant C_{T} .
$$

Proof. We use the flow interchange argument, introduced in 32, to find a stronger estimate as in [20, 29]. In other words, we perturb $\rho_{1, h}^{k}$ and $\rho_{2, h}^{k}$ by the heat flow. Let $\eta_{i}$ be the solution to

$$
\begin{cases}\partial_{t} \eta_{i}=\Delta \eta_{i} & \text { in }(0, T) \times \Omega \\ \nabla \eta_{i} \cdot \nu=0 & \text { in }(0, T) \times \partial \Omega \\ \eta_{i \mid t=0}=\rho_{i, h}^{k} . & \end{cases}
$$

Since the Entropy is geodesically convex then the heat flow is a 0 -flow of the Entropy $\mathcal{H}$, and satisfies the Evolution Variational Inequality, 3.5), see [23, 45, 3, 16, 44,

$$
\frac{1}{2}{\frac{d^{+}}{d \sigma}}_{\mid \sigma=s} W_{2}^{2}\left(\eta_{i}(s), \rho\right) \leqslant \mathcal{H}(\rho)-\mathcal{H}\left(\eta_{i}(s)\right)
$$

for all $s>0$ and $\rho \in \mathcal{P}_{2}^{\text {ac }}(\Omega)$.

Taking $\left(\eta_{1}(s), \eta_{2}(s)\right)$ as a competitor in the minimization 3.1, we get

$$
\sum_{i=1}^{2} \frac{1}{2} \frac{d^{+}}{d s} W_{2}^{2}\left(\eta_{i}(s), \rho_{i, h}^{k-1}\right)_{\mid s=0}+h \frac{d^{+}}{d s}\left(\sum_{i=1}^{2}\left(\mathcal{H}\left(\eta_{i}(s)\right)+\mathcal{V}_{i}\left(\eta_{i}(s)\right)\right)+\mathcal{F}_{m}\left(\eta_{1}(s)+\eta_{2}(s)\right)\right)_{\mid s=0} \geqslant 0
$$


Since $\eta_{i}(s)$ is a smooth positive function for $s>0$, the following computations are justified

$$
\begin{aligned}
\partial_{s}\left(\sum_{i=1}^{2}(\right. & \left.\left.\mathcal{H}\left(\eta_{i}(s)\right)+\mathcal{V}_{i}\left(\eta_{i}(s)\right)\right)+\mathcal{F}_{m}\left(\eta_{1}(s)+\eta_{2}(s)\right)\right) \\
& =\sum_{i=1}^{2}\left(\int_{\Omega} \Delta \eta_{i}(s)\left(\left(1+\log \left(\eta_{i}(s)\right)\right)+V_{i}\right)+\int_{\Omega} \Delta\left(\eta_{1}(s)+\eta_{2}(s)\right) F_{m}^{\prime}\left(\eta_{1}(s)+\eta_{2}(s)\right)\right. \\
& =-\sum_{i=1}^{2}\left(\int_{\Omega} \frac{\left|\nabla \eta_{i}(s)\right|^{2}}{\eta_{i}(s)}+\int_{\Omega} \nabla V_{i} \cdot \nabla \eta_{i}(s)\right)-\int_{\Omega}\left|\nabla\left(\eta_{1}(s)+\eta_{2}(s)\right)\right|^{2} F_{m}^{\prime \prime}\left(\eta_{1}(s)+\eta_{2}(s)\right) .
\end{aligned}
$$

In addition, Young's inequality gives

$$
-\int_{\Omega} \nabla V_{i}(s) \cdot \nabla \eta_{i} \leqslant \int_{\Omega}\left|\nabla V_{i}\right|\left|\nabla \eta_{i}(s)\right| \leqslant \frac{1}{2} \int_{\Omega}\left|\nabla V_{i}\right|^{2} \eta_{i}(s)+\frac{1}{2} \int_{\Omega} \frac{\left|\nabla \eta_{i}(s)\right|^{2}}{\eta_{i}(s)}
$$

Then, we have

$$
\begin{aligned}
& \partial_{s}\left(\sum_{i=1}^{2}\left(\mathcal{H}\left(\eta_{i}(s)\right)+\mathcal{V}_{i}\left(\eta_{i}(s)\right)\right)+\mathcal{F}_{m}\left(\eta_{1}(s)+\eta_{2}(s)\right)\right) \\
& \leqslant \sum_{i=1}^{2}\left(-\frac{1}{2} \int_{\Omega} \frac{\left|\nabla \eta_{i}(s)\right|^{2}}{\eta_{i}(s)}+\frac{1}{2} \int_{\Omega}\left|\nabla V_{i}\right|^{2} \eta_{i}(s)\right)-\int_{\Omega}\left|\nabla\left(\eta_{1}(s)+\eta_{2}(s)\right)\right|^{2} F_{m}^{\prime \prime}\left(\eta_{1}(s)+\eta_{2}(s)\right) .
\end{aligned}
$$

By definition of $F_{m}$, for $m \geqslant 1, F_{m}^{\prime \prime}(z)=m z^{m-2}$ for all $z \geqslant 0$ and, since $V_{i} \in W^{1, \infty}(\Omega)$,

$$
\begin{aligned}
\partial_{s}\left(\sum_{i=1}^{2}\left(\mathcal{H}\left(\eta_{i}(s)\right)+\mathcal{V}_{i}\left(\eta_{i}(s)\right)\right)\right. & \left.+\mathcal{F}_{m}\left(\eta_{1}(s)+\eta_{2}(s)\right)\right) \\
\leqslant & C-\frac{1}{2} \sum_{i=1}^{2} \int_{\Omega}\left|\nabla \eta_{i}(s)^{1 / 2}\right|^{2}-\frac{4}{m} \int_{\Omega}\left|\nabla\left(\eta_{1}(s)+\eta_{2}(s)\right)^{m / 2}\right|^{2}
\end{aligned}
$$

By a lower semi-continuity argument,

$$
\begin{aligned}
\frac{1}{2} \sum_{i=1}^{2} \int_{\Omega}\left|\nabla\left(\rho_{i, h}^{k}\right)^{1 / 2}\right|^{2}+\frac{4}{m} \int_{\Omega}\left|\nabla\left(\rho_{1, h}^{k}+\rho_{2, h}^{k}\right)^{m / 2}\right|^{2} & \\
& \leqslant C-\frac{d^{+}}{d s}\left(\sum_{i=1}^{2}\left(\mathcal{H}\left(\eta_{i}(s)\right)+\mathcal{V}_{i}\left(\eta_{i}(s)\right)\right)+\mathcal{F}_{m}\left(\eta_{1}(s)+\eta_{2}(s)\right)\right)_{\mid s=0} .
\end{aligned}
$$

Combining with 3.9 and 3.8 , we obtain

$$
h \sum_{i=1}^{2} \int_{\Omega}\left|\nabla\left(\rho_{i, h}^{k}\right)^{1 / 2}\right|^{2}+\frac{4 h}{m} \int_{\Omega}\left|\nabla\left(\rho_{1, h}^{k}+\rho_{2, h}^{k}\right)^{m / 2}\right|^{2} \leqslant \sum_{i=1}^{2}\left(\mathcal{H}\left(\rho_{i, h}^{k-1}\right)-\mathcal{H}\left(\rho_{i, h}^{k}\right)\right)+C h .
$$

Then summing over $k$, we obtain

$$
\left\|\rho_{1, h}^{1 / 2}\right\|_{L^{2}\left((0, T), H^{1}(\Omega)\right)}^{2}+\left\|\rho_{2, h}^{1 / 2}\right\|_{L^{2}\left((0, T), H^{1}(\Omega)\right)}^{2}+\frac{1}{m}\left\|\left(\rho_{1, h}+\rho_{2, h}\right)^{m / 2}\right\|_{L^{2}\left((0, T), H^{1}(\Omega)\right)}^{2} \leqslant C_{T},
$$

where we use the fact that $\left\|\rho_{i, h}^{1 / 2}\right\|_{L^{2}((0, T) \times \Omega)}^{2}=T$ and $\frac{1}{m}\left\|\left(\rho_{1, h}+\rho_{2, h}\right)^{m / 2}\right\|_{L^{2}((0, T) \times \Omega)}^{2} \leqslant C T$ by 3.3 .

Remark 3.5. The bound on $\left\|\rho_{i, h}^{1 / 2}\right\|_{L^{2}\left((0, T), H^{1}(\Omega)\right)}$ does not depend on $m$. However, if we multiply the Entropy $\mathcal{H}$ by a small parameter $\varepsilon>0$ in the JKO scheme (3.1), individual bounds blow up as $\varepsilon$ goes to 0 . 
Now we can deduce the following convergences.

Proposition 3.6. For all $T<+\infty$, there exist $\rho_{1}$ and $\rho_{2}$ in $\mathcal{C}^{0,1 / 2}\left([0, T], \mathcal{P}^{\text {ac }}(\Omega)\right)$ such that, up to a subsequence,

1. $\rho_{i, h}$ converges to $\rho_{i}$ in $L^{\infty}\left([0, T], \mathcal{P}^{\text {ac }}(\Omega)\right)$,

2. $\rho_{i, h}$ converges strongly to $\rho_{i}$ in $L^{1}((0, T) \times \Omega)$,

3. $\left(\rho_{1, h}+\rho_{2, h}\right)^{m / 2}$ converges strongly to $\left(\rho_{1}+\rho_{2}\right)^{m / 2}$ and $\nabla\left(\rho_{1, h}+\rho_{2, h}\right)^{m / 2}$ converges weakly to $\nabla\left(\rho_{1}+\rho_{2}\right)^{m / 2}$ in $L^{2}((0, T) \times \Omega)$.

Proof. 1. The first convergence is classical. We use the refined version of Ascoli-Arzelà's Theorem, [3. Proposition 3.3.1], and we immediately deduce that there exists a subsequence such that, for $i=1,2, \rho_{i, h}$ converges to $\rho_{i} \in \mathcal{C}^{1 / 2}\left([0, T], \mathcal{P}^{\text {ac }}(\Omega)\right)$ in $L^{\infty}\left([0, T], \mathcal{P}^{\text {ac }}(\Omega)\right)$.

The next two strong convergence results are obtained applying an extension of the AubinLions Lemma proved by Rossi and Savaré in [42, Theorem 2]. In the sequel, we work with the convergent subsequence obtained in the first step.

2. Let $\mathcal{G}: L^{1}(\Omega) \rightarrow(-\infty,+\infty]$ and $g: L^{1}(\Omega) \times L^{1}(\Omega) \rightarrow[0,+\infty]$ defined by

$$
\mathcal{G}(\rho):= \begin{cases}\left\|\rho^{1 / 2}\right\|_{H^{1}(\Omega)} & \text { if } \rho \in \mathcal{P}^{\mathrm{ac}}(\Omega) \text { and } \rho^{1 / 2} \in H^{1}(\Omega) \\ +\infty & \text { otherwise, }\end{cases}
$$

and

$$
g(\rho, \mu):= \begin{cases}W_{2}(\rho, \mu) & \text { if } \rho, \mu \in \mathcal{P}(\Omega) \\ +\infty & \text { otherwise }\end{cases}
$$

$\mathcal{G}$ is l.s.c and its sublevels are relatively compact in $L^{1}(\Omega)$ (see [20, 29]) and $g$ is a pseudodistance. According to (3.4) and (3.6), we have

$$
\sup _{h \leqslant 1} \int_{0}^{T} \mathcal{G}\left(\rho_{i, h}(t)\right) d t<+\infty, \text { and } \lim _{\tau \searrow 0} \sup _{h \leqslant 1} \int_{0}^{T-\tau} g\left(\rho_{i, h}(t+\tau), \rho_{i, h}(t)\right) d t=0,
$$

then applying Rossi-Savaré's Theorem, there exists a subsequence, not-relabeled, such that for $i=1,2, \rho_{i, h}$ converges in measure with respect to $t$ in $L^{1}(\Omega)$ to $\rho_{i}$. Moreover by Lebesgue's dominated convergence Theorem, $\rho_{i, h}$ converges to $\rho_{i}$ strongly in $L^{1}((0, T) \times \Omega)$.

3. With the same argument, we get a strong convergence on a nonlinear quantity of $\rho_{1, h}+\rho_{2, h}$. Let $\mathcal{G}$ define by

$$
\mathcal{G}(\rho):= \begin{cases}\left\|\rho^{m / 2}\right\|_{H^{1}(\Omega)} & \text { if } \rho \in \mathcal{P}^{\text {ac }}(\Omega) \text { and } \rho^{m / 2} \in H^{1}(\Omega) \\ +\infty & \text { otherwise, }\end{cases}
$$

and $g$ defined as before. We want to apply Theorem 2 of 42 in $L^{m}(\Omega)$ over the sequence $\frac{\rho_{1, h}+\rho_{2, h}}{2}$. By 3.6 , we obtain

$$
\sup _{h \leqslant 1} \int_{0}^{T} \mathcal{G}\left(\frac{\rho_{1, h}(t)+\rho_{2, h}(t)}{2}\right) d t<+\infty
$$

Since, it is well-known that for all $\rho_{1}, \rho_{2}, \mu_{1}, \mu_{2} \in \mathcal{P}^{\text {ac }}(\Omega)$,

$$
W_{2}^{2}\left(\frac{\rho_{1}+\rho_{2}}{2}, \frac{\mu_{1}+\mu_{2}}{2}\right) \leqslant \frac{1}{2} W_{2}^{2}\left(\rho_{1}, \mu_{1}\right)+\frac{1}{2} W_{2}^{2}\left(\rho_{2}, \mu_{2}\right)
$$

by 3.4 , we obtain

$$
\lim _{\tau \searrow 0} \sup _{h \leqslant 1} \int_{0}^{T-\tau} g\left(\frac{\rho_{1, h}+\rho_{2, h}}{2}(t+\tau), \frac{\rho_{1, h}+\rho_{2, h}}{2}(t)\right) d t=0 .
$$


Theorem 2 in [42] and Lebesgue's dominated convergence Theorem imply that $\rho_{1, h}+\rho_{2, h}$ converges strongly to $\rho_{1}+\rho_{2}$ in $L^{m}((0, T) \times \Omega)$. In addition, Krasnoselskii's Theorem, 17, Chapter 2], implies that $\left(\rho_{1, h}+\rho_{2, h}\right)^{m / 2}$ converges to $\left(\rho_{1}+\rho_{2}\right)^{m / 2}$ in $L^{2}((0, T) \times \Omega)$. To conclude, $\nabla\left(\rho_{1, h}+\rho_{2, h}\right)^{m / 2}$ is bounded in $L^{2}((0, T) \times \Omega)$, thanks to $(3.6)$, then $\nabla\left(\rho_{1, h}+\right.$ $\left.\rho_{2, h}\right)^{m / 2}$ weakly converges to $\nabla\left(\rho_{1}+\rho_{2}\right)^{m / 2}$ in $L^{2}((0, T) \times \Omega)$.

Remark 3.7. It is possible to obtain a strong convergence result in $L^{1}((0, T) \times \Omega)$ for the pressure $F_{m}^{\prime}\left(\rho_{1, h}+\rho_{2, h}\right)$. Indeed, since $\rho_{1, h}+\rho_{2, h}$ strongly converges in $L^{m}((0, T) \times \Omega)$, then up to a subsequence, $F_{m}^{\prime}\left(\rho_{1, h}+\rho_{2, h}\right) \rightarrow F_{m}^{\prime}\left(\rho_{1}+\rho_{2}\right)$ a.e. In addition using De La Vallée Poussin's Theorem, we show that $\left(F_{m}^{\prime}\left(\rho_{1, h}+\rho_{2, h}\right)\right)_{h}$ is uniformly integrable. We conclude applying Vitali's convergence Theorem.

Remark 3.8. Notice that we can drop one individual diffusion. Assume that we drop the individual Entropy in the JKO scheme (3.1) for one of the two densities, for instance $\rho_{2}$. The difficulty is to obtain a strong convergence for the sequence $\left(\rho_{2, h}\right)_{h}$. Proposition 3.6 gives the strong convergence of $\rho_{1, h}$ and $\rho_{1, h}+\rho_{2, h}$ in $L^{1}((0, T) \times \Omega)$ and $L^{m}((0, T) \times \Omega)$ respectively, and then pointwise on $(0, T) \times \Omega$. Consequently, $\rho_{2, h}=\left(\rho_{1, h}+\rho_{2, h}\right)-\rho_{1, h}$ converges pointwise on $(0, T) \times \Omega$. Moreover,

$$
\int_{0}^{T} \int_{\Omega} \rho_{2, h}(t, x)^{m} d x d t \leqslant \int_{0}^{T} \int_{\Omega}\left(\rho_{1, h}(t, x)+\rho_{2, h}(t, x)\right)^{m} d x d t \leqslant C_{T} .
$$

Then Vitali's convergence Theorem implies that $\rho_{2, h}$ strongly converges to $\rho_{2}$ in $L^{1}((0, T) \times \Omega)$.

\subsection{Existence of weak solutions to 1.3}

In this section, we start by giving the optimality conditions for 3.1). Instead of using horizontal perturbations, $\rho_{i, \varepsilon}=\Phi_{\varepsilon \#} \rho_{i, h}^{k+1}$, as introduced in [23] by Jordan, Kinderlherer and Otto, we will perturb $\rho_{i, h}^{k+1}$ with vertical perturbations introduced in [8, 11], and revisited in [43, 44], which consist in taking $\rho_{i, \varepsilon}=(1-\varepsilon) \rho_{i, h}^{k+1}+\varepsilon \tilde{\rho}_{i}$, for any $\tilde{\rho}_{i} \in L^{\infty}(\Omega)$. Before giving the optimality conditions for (3.1), we state the following Lemma.

Lemma 3.9. For all $k \geqslant 1, \rho_{i, h}^{k}>0$ a.e. and $\log \left(\rho_{i, h}^{k}\right) \in L^{1}(\Omega)$.

Proof. The proof is the same as [44, Lemma 8.6].

This Lemma ensures the uniqueness (up to a constant) of the Kantorovich potential in the transport from $\rho_{i, h}^{k+1}$ to $\rho_{i, h}^{k}$ and then, we can easily compute the first variation of $W_{2}\left(\cdot, \rho_{i, h}^{k}\right)$ according to [44, Proposition 7.17].

Proposition 3.10. For $i \in\{1,2\}, \rho_{i, h}^{k+1}$ satisfies

$$
\nabla V_{i}+\nabla \log \left(\rho_{i, h}^{k+1}\right)+\nabla F_{m}^{\prime}\left(\rho_{1, h}^{k+1}+\rho_{2, h}^{k+1}\right)+\frac{\nabla \varphi_{i, h}^{k+1}}{h}=0 \quad \rho_{i, h}^{k+1}-\text { a.e },
$$

where $\varphi_{i, h}^{k+1}$ is the (unique) Kantorovich potential from $\rho_{i, h}^{k+1}$ to $\rho_{i, h}^{k}$.

Proof. The proof is a straightforward adaptation of classical result, see for instance [44].

A classical consequence of the previous Proposition is that $\rho_{1, h}$ and $\rho_{2, h}$ are solutions to a discrete approximation of system 1.3 . 
Proposition 3.11. Let $h>0$, for all $T>0$, let $N$ such that $N=\left\lfloor\frac{T}{h}\right\rfloor$. Then for all $\left(\phi_{1}, \phi_{2}\right) \in$ $\mathcal{C}_{c}^{\infty}\left([0, T) \times \mathbb{R}^{n}\right)^{2}$ and for all $i \in\{1,2\}$,

$$
\begin{aligned}
\int_{0}^{T} & \int_{\Omega} \rho_{i, h}(t, x) \partial_{t} \phi_{i}(t, x) d x d t+\int_{\Omega} \rho_{i, 0}(x) \phi_{i}(0, x) d x \\
& =h \sum_{k=0}^{N-1} \int_{\Omega} \nabla V_{i}(x) \cdot \nabla \phi_{i}\left(t_{k}, x\right) \rho_{i, h}^{k+1}(x) d x+h \sum_{k=0}^{N-1} \int_{\Omega} \nabla \rho_{i, h}^{k+1}(x) \cdot \nabla \phi_{i}\left(t_{k}, x\right) d x \\
& +h \sum_{k=0}^{N-1} \int_{\Omega} \nabla F_{m}^{\prime}\left(\rho_{1, h}^{k+1}+\rho_{2, h}^{k+1}\right) \cdot \nabla \phi_{i}\left(t_{k}, x\right) \rho_{i, h}^{k+1}(x) d x+\sum_{k=0}^{N-1} \int_{\Omega \times \Omega} \mathcal{R}\left[\phi_{i}\left(t_{k}, \cdot\right)\right](x, y) d \gamma_{i, h}^{k}(x, y)
\end{aligned}
$$

where $t_{k}=h k\left(t_{N}:=T\right)$ and $\gamma_{i, h}^{k}$ is the optimal transport plan in $W_{2}\left(\rho_{i, h}^{k}, \rho_{i, h}^{k+1}\right)$. Moreover, $\mathcal{R}$ is defined such that, for all $\phi \in \mathcal{C}_{c}^{\infty}\left([0, T) \times \mathbb{R}^{n}\right)$,

$$
|\mathcal{R}[\phi](x, y)| \leqslant \frac{1}{2}\left\|D^{2} \phi\right\|_{L^{\infty}\left([0, T) \times \mathbb{R}^{n}\right)}|x-y|^{2} .
$$

Proof. We multiply by $\rho_{i, h}^{k+1}$ and take the $L^{2}$-inner product between the 1.h.s. of (3.13) and $\nabla \phi_{i}\left(t_{k}, \cdot\right)$, for any $\phi_{i} \in \mathcal{C}_{c}^{\infty}\left([0, T) \times \mathbb{R}^{n}\right)$ and the proof is the same as in [1, 29], for example.

Another consequence of $(3.13)$ is an improvment of the regularity of $\rho_{i, h}$.

Proposition 3.12. For all $T>0$ and $i=1,2$, we have

- $\left(\rho_{1, h}+\rho_{2, h}\right)^{1 / 2} \nabla F_{m}^{\prime}\left(\rho_{1, h}+\rho_{2, h}\right)$ is bounded in $L^{2}((0, T) \times \Omega)$,

- $\rho_{i, h}, \rho_{1, h}+\rho_{2, h}$ are bounded in $L^{2 m-1}(((0, T) \times \Omega)$,

- $\nabla F_{m}^{\prime}\left(\rho_{1, h}+\rho_{2, h}\right) \rho_{i, h}$ is bounded in $L^{2-1 / m}((0, T) \times \Omega)$ and $\rho_{i, h}$ is bounded in $L^{2-1 / m}\left((0, T), W^{1,2-1 / m}(\Omega)\right)$.

Proof. The first item is a direct consequence of (3.13), using Proposition 3.6. see for example [26], and by Poincaré-Wirtinger inequality, we prove the second item. Now we will prove the third item. The first part is straightforward applying Hölder's inequality,

$$
\left\|\nabla F_{m}^{\prime}\left(\rho_{1, h}+\rho_{2, h}\right) \rho_{i, h}\right\|_{L^{2-1 / m}} \leqslant\left\|\nabla F_{m}^{\prime}\left(\rho_{1, h}+\rho_{2, h}\right) \rho_{i, h}^{1 / 2}\right\|_{L^{2}}^{1-1 / 2 m}\left\|\rho_{i, h}\right\|_{L^{2 m-1}}^{1 / 2 m}<+\infty .
$$

According to 3.13, we obtain a.e.

$$
\left|\nabla \rho_{i, h}^{k+1}\right|^{2-1 / m} \leqslant C\left(\left|\frac{\nabla \varphi_{i, h}^{k+1} \rho_{i, h}^{k+1}}{h}\right|^{2-1 / m}+\left(\left|\nabla V_{i}\right| \rho_{i, h}^{k+1}\right)^{2-1 / m}+\left(\left|\nabla F_{m}^{\prime}\left(\rho_{1, h}^{k+1}+\rho_{2, h}^{k+1}\right)\right| \rho_{i, h}^{k+1}\right)^{2-1 / m}\right) .
$$

We have already seen that $\nabla F_{m}^{\prime}\left(\rho_{1, h}+\rho_{2, h}\right) \rho_{i, h}$ is bounded in $L^{2-1 / m}((0, T) \times \Omega)$ and since $\rho_{i, h} \in$ $L^{1} \cap L^{2 m-1}((0, T) \times \Omega),\left\|\nabla V_{i} \rho_{i, h}\right\|_{L^{2-1 / m}} \leqslant C$. To deal with the last term, notice that by Hölder's inequality,

$$
\int_{\Omega}\left|\frac{\nabla \varphi_{i, h}^{k+1} \rho_{i, h}^{k+1}}{h}\right|^{2-1 / m} \leqslant \frac{1}{h^{2-1 / m}} W_{2}\left(\rho_{i, h}^{k}, \rho_{i, h}^{k+1}\right)^{2-1 / m}\left\|\rho_{i, h}^{k+1}\right\|_{L^{2 m-1}}^{(2 m-1) / 2 m}
$$

and then,

$$
\begin{aligned}
h \sum_{k=0}^{N-1} \int_{\Omega}\left|\frac{\nabla \varphi_{i, h}^{k+1} \rho_{i, h}^{k+1}}{h}\right|^{2-1 / m} & \leqslant C h^{1 / m-1} N^{1 / 2 m}\left(\sum_{k=0}^{N-1} W_{2}^{2}\left(\rho_{i, h}^{k}, \rho_{i, h}^{k+1}\right)\right)^{(2 m-1) / 2 m} \\
& \leqslant C T^{1 / 2 m}\left(\frac{\sum_{k=0}^{N-1} W_{2}^{2}\left(\rho_{i, h}^{k}, \rho_{i, h}^{k+1}\right)}{h}\right)^{(2 m-1) / 2 m} \\
& \leqslant C_{T},
\end{aligned}
$$

by 3.2 where $T=N h$. Then $\nabla \rho_{i, h}$ is bounded in $L^{2-1 / m}((0, T) \times \Omega)$ and we conclude the proof with Poincaré-Wirtinger inequality. 
Now we are able to prove Theorem 2.2

Proof of Theorem 2.2. We have to pass to the limit in all terms in Proposition 3.11 as $h \searrow 0$. The remainder term converges to 0 using the total square distance estimate (3.4) and the linear term converges to

$$
\int_{0}^{T} \int_{\Omega} \rho_{i} \partial_{t} \phi_{i}-\int_{0}^{T} \int_{\Omega} \nabla V_{i} \cdot \nabla \phi_{i} \rho_{i},
$$

when $h$ goes to 0 thanks to Proposition 3.6

Furthermore, since $\nabla \rho_{i, h}$ is bounded in $L^{2-1 / m}((0, T) \times \Omega)$, because of Proposition 3.12 and the fact that $\rho_{i, h}$ strongly converges to $\rho_{i}$ in $L^{1}((0, T) \times \Omega)$, we conclude that $\nabla \rho_{i, h}$ converges weakly to $\nabla \rho_{i}$ in $L^{2-1 / m}((0, T) \times \Omega)$. This implies that the individual diffusion term converges to

$$
\int_{0}^{T} \int_{\Omega} \nabla \phi_{i} \cdot \nabla \rho_{i} d x d t
$$

It remains to study the convergence of the nonlinear cross diffusion term. First, we remark that $\nabla F_{m}^{\prime}\left(\rho_{1, h}^{k+1}+\rho_{2, h}^{k+1}\right)$ can be rewritten as

$$
\nabla F_{m}^{\prime}\left(\rho_{1, h}^{k+1}+\rho_{2, h}^{k+1}\right)=2 \frac{\left(\rho_{1, h}^{k+1}+\rho_{2, h}^{k+1}\right)^{m / 2}}{\rho_{1, h}^{k+1}+\rho_{2, h}^{k+1}} \nabla\left(\rho_{1, h}^{k+1}+\rho_{2, h}^{k+1}\right)^{m / 2}
$$

Then

$$
\nabla F_{m}^{\prime}\left(\rho_{1, h}^{k+1}+\rho_{2, h}^{k+1}\right) \rho_{i, h}^{k+1}=2 G_{1-m / 2}\left(\rho_{1, h}^{k+1}, \rho_{2, h}^{k+1}\right) \nabla\left(\rho_{1, h}^{k+1}+\rho_{2, h}^{k+1}\right)^{m / 2},
$$

where $G_{\alpha}: \mathbb{R}^{+} \times \mathbb{R}^{+} \rightarrow \mathbb{R}$ is the continuous function (for $\alpha<1$ ) defined by

$$
G_{\alpha}(x, y):= \begin{cases}\frac{x}{(x+y)^{\alpha}} & \text { if } x>0, y \geqslant 0 \\ 0 & \text { otherwise. }\end{cases}
$$

As $m \geqslant 1,1-\frac{m}{2}<1$ so $G_{1-m / 2}$ is continuous and since, up to a subsequence, $\rho_{i, h}$ converges to $\rho_{i}$ a.e., we obtain that $G_{1-m / 2}\left(\rho_{1, h}, \rho_{2, h}\right)$ converges to $G_{1-m / 2}\left(\rho_{1}, \rho_{2}\right)$ a.e. in $(0, T) \times \Omega$. In addition,

$$
\left|G_{1-m / 2}\left(\rho_{1, h}, \rho_{2, h}\right)\right|=\left|\left(\rho_{1, h}+\rho_{2, h}\right)^{m / 2} \frac{\rho_{1, h}}{\rho_{1, h}+\rho_{2, h}}\right| \leqslant\left(\rho_{1, h}+\rho_{2, h}\right)^{m / 2} .
$$

Up to a subsequence, $\rho_{i, h}$ and $\rho_{1, h}+\rho_{2, h}$ converge a.e. in $(0, T) \times \Omega$, and, since $\left(\rho_{1, h}+\rho_{2, h}\right)^{m / 2}$ converges to $\left(\rho_{1}+\rho_{2}\right)^{m / 2}$ in $L^{2}((0, T) \times \Omega)$, there exists a function $g \in L^{2}((0, T) \times \Omega)$ such that,

$$
\left|\left(\rho_{1, h}+\rho_{2, h}\right)^{m / 2}\right| \leqslant g .
$$

Then Lebesgue's dominated convergence Theorem implies that $G_{1-m / 2}\left(\rho_{1, h}, \rho_{2, h}\right)$ converges strongly in $L^{2}((0, T) \times \Omega)$ to $G_{1-m / 2}\left(\rho_{1}, \rho_{2}\right)$. Moreover, $\nabla\left(\rho_{1, h}^{k+1}+\rho_{2, h}^{k+1}\right)^{m / 2}$ converges weakly in $L^{2}((0, T) \times$ $\Omega$ ), by Proposition 3.6 , then $\nabla F_{m}^{\prime}\left(\rho_{1, h}+\rho_{2, h}\right) \rho_{i, h}$ converges weakly in $L^{1}((0, T) \times \Omega)$ to $\nabla F_{m}^{\prime}\left(\rho_{1}+\right.$ $\left.\rho_{2}\right) \rho_{i}$ and

$$
h \sum_{k=0}^{N-1} \int_{\Omega} \nabla F_{m}^{\prime}\left(\rho_{1, h}^{k+1}+\rho_{2, h}^{k+1}\right) \cdot \nabla \phi_{i}\left(t_{k}, x\right) \rho_{i, h}^{k+1}(x) d x \rightarrow \int_{0}^{T} \int_{\Omega} \nabla F_{m}^{\prime}\left(\rho_{1}+\rho_{2}\right) \cdot \nabla \phi_{i} \rho_{i} d x d t .
$$

In addition, by Proposition 3.12 , we obtain that $\nabla F_{m}^{\prime}\left(\rho_{1}+\rho_{2}\right) \rho_{i} \in L^{2-1 / m}((0, T) \times \Omega)$, which concludes the proof. 


\section{Coupling by hard congestion}

In this section we prove the existence of a weak solution to 1.1p, i.e. Theorem 2.3 This system can be seen as gradient flow in a Wasserstein product space. Using the Jordan-Kinderlherer-Otto scheme, we construct two sequences defined in the following way: let $h>0$ be a time step, we construct a sequence $\left(\rho_{1, h}^{k}, \rho_{2, h}^{k}\right)$ with $\left(\rho_{1, h}^{0}, \rho_{2, h}^{0}\right)=\left(\rho_{1,0}, \rho_{2,0}\right)$ and $\left(\rho_{1, h}^{k+1}, \rho_{2, h}^{k+1}\right)$ is a solution to

$$
\inf _{\left(\rho_{1}, \rho_{2}\right) \in \mathcal{K}} \sum_{i=1}^{2}\left[\frac{1}{2 h} W_{2}^{2}\left(\rho_{i}, \rho_{i, h}^{k}\right)+\mathcal{H}\left(\rho_{i}\right)+\mathcal{V}_{i}\left(\rho_{i}\right)\right]
$$

where $\mathcal{K}:=\left\{\left(\rho_{1}, \rho_{2}\right) \in \mathcal{P}^{\mathrm{ac}}(\Omega)^{2}: \rho_{1}+\rho_{2} \leqslant 1\right\}$ and $|\Omega|>2$. The direct method shows that these sequences are well-defined. As before, we define the piecewise constant interpolations $\rho_{i, h}: \mathbb{R}^{+} \rightarrow$ $\mathcal{P}^{\mathrm{ac}}(\Omega)$ by

$$
\rho_{i, h}(t):=\rho_{i, h}^{k+1}, \quad \text { if } t \in(k h,(k+1) h] .
$$

\subsection{Estimates and convergences}

In the following proposition, we list the classical estimates coming from the Wasserstein gradient flow theory.

Proposition 4.1. Let $T>0$. Then there exists $C>0$ such that for $i \in\{1,2\}$ and for all $k \geqslant 0$ such that $k \leqslant N:=\left\lfloor\frac{T}{h}\right\rfloor$,

$$
\rho_{1, h}^{k}+\rho_{2, h}^{k} \leqslant 1, \quad \mathcal{H}\left(\rho_{i, h}^{k}\right) \leqslant C, \quad \sum_{k=0}^{N-1} W_{2}^{2}\left(\rho_{i, h}^{k}, \rho_{i, h}^{k+1}\right) \leqslant C h .
$$

As in the previous section, we need stronger estimates in order to handle the very degenerate cross diffusion term, $\operatorname{div}\left(\rho_{i} \nabla p\right)$.

Proposition 4.2. For all $T>0$, there exists a constant $C_{T}>0$ such that

$$
\left\|\rho_{1, h}^{1 / 2}\right\|_{L^{2}\left((0, T), H^{1}(\Omega)\right)}+\left\|\rho_{2, h}^{1 / 2}\right\|_{L^{2}\left((0, T), H^{1}(\Omega)\right)} \leqslant C_{T} .
$$

Proof. We apply the flow interchange technique as previously, Proposition 3.4 Keeping the same notations as in the previous section, we denote by $\eta_{i}$ the heat flow with initial condition $\rho_{i, h}^{k}$. Since the heat flow decreases the $L^{\infty}$-norm, $\left(\eta_{1}(s), \eta_{2}(s)\right)$, defined in (3.7), is admissible for the minimization problem (4.1), for all $s \geqslant 0$. Then the same computations as in Proposition 3.4 give the result.

Consequently, we deduce the following convergences.

Proposition 4.3. For all $T>0$, there exist $\rho_{1}$ and $\rho_{2}$ in $\mathcal{C}^{0,1 / 2}\left([0, T], \mathcal{P}^{\mathrm{ac}}(\Omega)\right)$ such that, up to a subsequence,

1. $\rho_{i, h}$ converges to $\rho_{i}$ in $L^{\infty}\left([0, T], \mathcal{P}^{\mathrm{ac}}(\Omega)\right)$,

2. $\rho_{i, h}$ converges strongly to $\rho_{i}$ in $L^{p}((0, T) \times \Omega)$, for all $p \in[1,+\infty)$ and $\nabla \rho_{i, h}$ converges narrowly to $\nabla \rho_{i}$.

Proof. The total square distance estimate (4.2) and the refined version of Ascoli-Arzelà's Theorem, [3. Proposition 3.3.1], implies that $\rho_{i, h}$ converges to $\rho_{i} \in \mathcal{C}^{1 / 2}\left([0, T], \mathcal{P}^{\text {ac }}(\Omega)\right)$ in $L^{\infty}\left([0, T], \mathcal{P}^{\text {ac }}(\Omega)\right)$. As in Proposition 3.6, applying [42, Theorem 2], we obtain that $\rho_{i, h}$ converges strongly to $\rho_{i}$ in $L^{1}((0, T) \times \Omega)$. And noticing that $\rho_{i, h}, \rho_{i} \leqslant 1$ a.e., we deduce that the strong convergence holds in $L^{p}((0, T) \times \Omega)$, for all $p \in[1,+\infty)$. To conclude, we remark that $\nabla \rho_{i, h}=2 \rho_{i, h}^{1 / 2} \nabla \rho_{i, h}^{1 / 2}, \rho_{i, h}^{1 / 2}$ strongly converges to $\rho_{i}^{1 / 2}$ in $L^{2}((0, T) \times \Omega)$ and $\nabla \rho_{i, h}^{1 / 2}$ weakly converges to $\nabla \rho_{i}^{1 / 2}$ in $L^{2}((0, T) \times \Omega)$.

We end this section by a lemma implying the uniqueness of the pair of Kantorovich potentials from $\rho_{i, h}^{k+1}$ to $\rho_{i, h}^{k}$ and then the existence of the first variation of $W_{2}^{2}\left(\cdot, \rho_{i, h}^{k}\right)$ (Propositions 7.18 and 7.17 from [44). 
Lemma 4.4. Minimizers of 4.1) satisfy $\rho_{i, h}^{k}>0$ a.e. and $\log \left(\rho_{i, h}^{k}\right) \in L^{1}(\Omega)$.

Proof. The proof is the same as in [44, Lemma 8.5]. Indeed we can use a constant perturbation $\tilde{\rho}$ because $(\tilde{\rho}, \tilde{\rho})$ is admissible in 4.1$)(\tilde{\rho}+\tilde{\rho}=2 /|\Omega| \leqslant 1$ by 1.2$)$.

\subsection{Pressure field associated to the constraint}

In this section, we introduce a discrete pressure associated to the constraint $\rho_{1, h}^{k+1}+\rho_{2, h}^{k+1} \leqslant 1$. This common pressure is obtained arguing as in [33] in the case of one population.

Lemma 4.5. Let $\left(\rho_{1, h}^{k+1}, \rho_{2, h}^{k+1}\right)$ be the unique solution to 4.1). Then for all $\left(\rho_{1}, \rho_{2}\right) \in \mathcal{K}$,

$$
\int_{\Omega} \psi_{1, h}^{k+1}\left(\rho_{1}-\rho_{1, h}^{k+1}\right)+\int_{\Omega} \psi_{2, h}^{k+1}\left(\rho_{2}-\rho_{2, h}^{k+1}\right) \geqslant 0,
$$

where $\psi_{i, h}^{k+1}=\frac{\varphi_{i, h}^{k+1}}{h}+V_{i}+1+\log \left(\rho_{i, h}^{k+1}\right)$ and $\varphi_{i, h}^{k+1}$ is the optimal (up to a constant) Kantorovich potential in $W_{2}\left(\rho_{i, h}^{k+1}, \rho_{i, h}^{k}\right)$.

Proof. The proof of this result is the same as Lemma 3.1 in [33].

Remark 4.6. Notice that 4.4 can be rewritten as

$$
\int_{\Omega} \psi_{1, h}^{k} f_{1}+\int_{\Omega} \psi_{2, h}^{k} f_{2} \geqslant 0,
$$

for all functions $f_{1}, f_{2} \in L^{\infty}(\Omega)$ such that

$$
f_{1}+f_{2} \leqslant \frac{1-\rho_{1, h}^{k}-\rho_{2, h}^{k}}{\varepsilon}, \quad f_{i} \geqslant \frac{-\rho_{i, h}^{k}}{\varepsilon} \text { and } \int_{\Omega} f_{i}=0,
$$

for all $0<\varepsilon \ll 1$.

In the next proposition, we introduce the common discrete pressure.

Proposition 4.7. There exists $p_{h}^{k} \geqslant 0$ such that for all, $k \geqslant 1$,

$$
p_{h}^{k}\left(1-\rho_{1, h}^{k}-\rho_{2, h}^{k}\right)=0 \quad \text { a.e. }
$$

In addition, $p_{h}^{k}$ satisfies

$$
\nabla p_{h}^{k}=-\frac{\nabla \varphi_{i, h}^{k}}{h}-\nabla V_{i}-\nabla \log \left(\rho_{i, h}^{k}\right) \quad \text { a.e },
$$

for $i=1,2$.

Proof. Let $S:=\left\{\rho_{1, h}^{k}+\rho_{2, h}^{k}=1\right\}$ be the set where the constraint is saturated. Firstly, we choose $f_{2}=0$ on $\Omega$ and $f_{1}=0$ on $S$ in Remark 4.6. Then we have

$$
\int_{S^{c}} \psi_{1, h}^{k} f_{1} \geqslant 0,
$$

for all $f_{1} \in L^{\infty}(\Omega)$. This implies that there exists a constant $C_{1}$ such that $\psi_{1, h}^{k}=C_{1}$ a.e. on $S^{c}$. Applying the same argument with $f_{1}=0$ on $\Omega$ and $f_{2}=0$ on $S$, we find a constant $C_{2}$ such that $\psi_{2, h}^{k}=C_{2}$ a.e. on $S^{c}$. And since $f_{1}$ and $f_{2}$ satisfy 4.5 , we have

$$
\int_{\Omega}\left(\psi_{1, h}^{k}-C_{1}\right) f_{1}+\int_{\Omega}\left(\psi_{2, h}^{k}-C_{2}\right) f_{2} \geqslant 0 .
$$

Now, choosing $f_{1}=f$ and $f_{2}=-f$ on $S$ and by symmetry $\left(f_{1}=-f\right.$ and $\left.f_{2}=f\right)$, we find

$$
\int_{S}\left(\left(\psi_{1, h}^{k}-C_{1}\right)-\left(\psi_{2, h}^{k}-C_{2}\right)\right) f=0,
$$


for all $f \in L^{\infty}(\Omega)$. We conclude that $\left(\psi_{1, h}^{k}-C_{1}\right)=\left(\psi_{2, h}^{k}-C_{2}\right)=: \psi_{h}^{k}$ a.e. on $S$ and consequently

$$
\int_{S} \psi_{h}^{k}\left(f_{1}+f_{2}\right) \geqslant 0
$$

On the other hand, since $f_{1}+f_{2} \leqslant 0$ on $S, \psi_{h}^{k} \leqslant 0$ a.e on $S$, then we define $p_{h}^{k}$ by

$$
p_{h}^{k}:=\left(C_{1}-\psi_{1, h}^{k}\right)_{+}=\left(C_{2}-\psi_{2, h}^{k}\right)_{+} .
$$

By definition, we have $p_{h}^{k}\left(1-\rho_{1, h}^{k}-\rho_{2, h}^{k}\right)=0$ a.e. and since $\psi_{i, h}^{k}$ is differentiable a.e., the proof is completed.

Now, we define the piecewise interpolation $p_{h}: \mathbb{R}^{+} \rightarrow L^{1}(\Omega)$ by

$$
p_{h}(t):=p_{h}^{k+1}, \quad \text { if } t \in(k h,(k+1) h] .
$$

Notice that $p_{h}(t) \geqslant 0$ and for all $t \geqslant 0, p_{h}(t)\left(1-\rho_{1, h}(t)-\rho_{2, h}(t)\right)=0$ a.e. Therefore, we immediately deduce the following estimate on the pressure.

Proposition 4.8. For all $T>0, p_{h}$ is bounded in $L^{2}\left((0, T), H^{1}(\Omega)\right)$.

Proof. First, we prove that $\nabla p_{h}$ is bounded in $L^{2}((0, T) \times \Omega)$ and then we will conclude using Poincaré's inequality. By definition of $p_{h}^{k+1}$, we have

$$
\begin{aligned}
\int_{\Omega}\left|\nabla p_{h}^{k+1}\right|^{2}\left(\rho_{1, h}^{k+1}+\rho_{2, h}^{k+1}\right) & =\sum_{i=1}^{2} \int_{\Omega}\left|\nabla \psi_{i, h}^{k+1}\right|^{2} \rho_{i, h}^{k+1} \\
& \leqslant C \sum_{i=1}^{2}\left(\int_{\Omega}\left|\frac{\nabla \phi_{i, h}^{k+1}}{h}\right|^{2} \rho_{i, h}^{k+1}+\int_{\Omega}\left|\nabla V_{i}\right|^{2} \rho_{i, h}^{k+1}+\int_{\Omega} \frac{\left|\nabla \rho_{i, h}^{k+1}\right|^{2}}{\rho_{i, h}^{k+1}}\right) \\
& \leqslant C \sum_{i=1}^{2}\left(\frac{1}{h^{2}} W_{2}^{2}\left(\rho_{i, h}^{k}, \rho_{i, h}^{k+1}\right)+C+\left\|\left(\rho_{i, h}^{k+1}\right)^{1 / 2}\right\|_{H^{1}(\Omega)}\right),
\end{aligned}
$$

where the last line is obtained using the fact that $\nabla V_{i} \in L^{\infty}(\Omega)$. Summing the previous inequalities over $k$ and by 4.1 and 4.3 , we obtain that

$$
\int_{0}^{T} \int_{\Omega}\left|\nabla p_{h}(t)\right|^{2}\left(\rho_{1, h}(t)+\rho_{2, h}(t)\right) \leqslant C .
$$

Since $p_{h}(t)=0$ a.e. on $\left\{\rho_{1, h}(t)+\rho_{2, h}(t)<1\right\}$, we deduce

$$
\int_{0}^{T} \int_{\Omega}\left|\nabla p_{h}(t)\right|^{2}=\int_{0}^{T} \int_{\Omega}\left|\nabla p_{h}(t)\right|^{2}\left(\rho_{1, h}(t)+\rho_{2, h}(t)\right) \leqslant C .
$$

We conclude with the same argument as 38. Using Poincaré's inequality, since $\left|\left\{p_{h}(t)=0\right\}\right| \geqslant$ $\left|\left\{\rho_{1, h}(t)+\rho_{2, h}(t)<1\right\}\right| \geqslant|\Omega|-2>0$, by 1.2 , we obtain that $p_{h}$ is bounded in $L^{2}\left((0, T), H^{1}(\Omega)\right)$.

Using Proposition 4.8, the regularity of $\rho_{i}$ can be improved.

Corollary 4.9. For all $T>0$ and $i=1,2, \rho_{i, h}$ is bounded in $L^{2}\left((0, T), H^{1}(\Omega)\right)$.

Proof. By 4.6 combined with $\rho_{i, h}^{k+1} \leqslant 1$, we obtain that

$$
\left|\nabla \rho_{i, h}^{k+1}\right|^{2} \leqslant C\left(\frac{\left|\nabla \varphi_{i, h}^{k+1}\right|^{2}}{h^{2}} \rho_{i, h}^{k+1}+\left|\nabla V_{i}\right|^{2} \rho_{i, h}^{k+1}+\left|\nabla p_{h}^{k+1}\right|^{2}\right) \quad \text { a.e. }
$$

Since, by Proposition $4.8, \nabla p_{h}$ is bounded in $L^{2}((0, T) \times \Omega)$ and

$$
h \sum_{k=0}^{N-1} \int_{\Omega} \frac{\left|\nabla \varphi_{i, h}^{k+1}\right|^{2}}{h^{2}} \rho_{i, h}^{k+1} \leqslant C,
$$


because of 4.1), we have

$$
\left\|\nabla \rho_{i, h}\right\|_{L^{2}((0, T) \times \Omega)} \leqslant C .
$$

The proof is concluded noticing that

$$
\left\|\rho_{i, h}\right\|_{L^{2}((0, T) \times \Omega)} \leqslant\left\|\rho_{i, h}\right\|_{L^{\infty}((0, T) \times \Omega)}^{1 / 2}\left\|\rho_{i, h}\right\|_{L^{1}((0, T) \times \Omega)}^{1 / 2} \leqslant T^{1 / 2} .
$$

To analyse the pressure field $p_{h}$, we recall the following lemma, 33, 38,

Lemma 4.10. [38, Lemma 3.5] Let $\left(p_{h}\right)_{h>0}$ be a bounded sequence in $L^{2}\left([0, T], H^{1}(\Omega)\right)$ and $\left(\rho_{h}\right)_{h>0}$ a sequence of piecewise constant curves valued in $\mathcal{P}(\Omega)$ which satisfiy $W_{2}\left(\rho_{h}(t), \rho_{h}(s)\right) \leqslant$ $C \sqrt{t-s-h}$ for all $s<t \in[0, T]$ and $\rho_{h} \leqslant C$ for a fixed constant $C$. Suppose that

$$
p_{h} \geqslant 0, \quad p_{h}\left(1-\rho_{h}\right)=0, \quad \rho_{h} \leqslant 1,
$$

and that

$$
p_{h} \rightarrow p \text { weakly in } L^{2}\left([0, T], H^{1}(\Omega)\right) \text { and } \rho_{h} \rightarrow \rho \text { uniformly in } \mathcal{P}(\Omega) .
$$

Then $p(1-\rho)=0$.

Consequently, one has

Proposition 4.11. There exists $p \in L^{2}\left([0, T], H^{1}(\Omega)\right)$ such that $p_{h}$ converges weakly in $L^{2}\left([0, T], H^{1}(\Omega)\right)$ to $p$, where $p$ satisfies

$$
p \geqslant 0, \quad p\left(1-\rho_{1}-\rho_{2}\right)=0, \quad \rho_{1}+\rho_{2} \leqslant 1 \text { a.e. in }[0, T] \times \Omega .
$$

In addition, $\rho_{i, h} \nabla p_{h}$ narrowly converges to $\rho_{i} \nabla p$.

Proof. We apply Lemma 4.10 to $\rho_{h}:=\rho_{1, h}+\rho_{2, h}$ and $p_{h}$. According to Proposition 4.8 , $p_{h}$ weakly converges in $L^{2}\left((0, T), H^{\mathrm{T}}(\Omega)\right)$ to $p$ such that

$$
p \geqslant 0, \quad p\left(1-\rho_{1}-\rho_{2}\right)=0, \quad \rho_{1}+\rho_{2} \leqslant 1 .
$$

Moreover, using the estimate on $p_{h}$, we know that $\nabla p_{h}$ weakly converges to $\nabla p$ in $L^{2}((0, T) \times$ $\Omega$ ). Then since $\rho_{i, h}$ strongly converges to $\rho_{i}$ in $L^{2}((0, T) \times \Omega)$ (Proposition 4.3 ), by strong-weak convergence, we obtain that $\rho_{i, h} \nabla p_{h}$ narrowly converges to $\rho_{i} \nabla p$.

\subsection{Existence of weak solutions to (1.1)}

Arguing as in Proposition 3.11, $\left(\rho_{1, h}, \rho_{2, h}\right)$ is solution to a discrete approximation of system (1.1).

Proposition 4.12. Let $h>0$, for all $T>0$, let $N$ such that $N=\left\lfloor\frac{T}{h}\right\rfloor$. Then for all $\left(\phi_{1}, \phi_{2}\right) \in$ $\mathcal{C}_{c}^{\infty}\left([0, T) \times \mathbb{R}^{n}\right)^{2}$ and for all $i \in\{1,2\}$,

$$
\begin{aligned}
\int_{0}^{T} \int_{\Omega} \rho_{i, h}(t, x) \partial_{t} \phi_{i}(t, x) d x d t+\int_{\Omega} \rho_{i, 0}(x) \phi_{i}(0, x) d x & \\
= & h \sum_{k=0}^{N-1} \int_{\Omega} \nabla V_{i}(x) \cdot \nabla \phi_{i}\left(t_{k}, x\right) \rho_{i, h}^{k+1}(x) d x+h \sum_{k=0}^{N-1} \int_{\Omega} \nabla \rho_{i, h}^{k+1}(x) \cdot \nabla \phi_{i}\left(t_{k}, x\right) d x \\
& +h \sum_{k=0}^{N-1} \int_{\Omega} \nabla p_{h}^{k+1} \cdot \nabla \phi_{i}\left(t_{k}, x\right) \rho_{i, h}^{k+1}(x) d x+\sum_{k=0}^{N-1} \int_{\Omega \times \Omega} \mathcal{R}\left[\phi_{i}\left(t_{k}, \cdot\right)\right](x, y) d \gamma_{i, h}^{k}(x, y)
\end{aligned}
$$

where $t_{k}=h k\left(t_{N}:=T\right)$ and $\gamma_{i, h}^{k}$ is the optimal transport plan in $W_{2}\left(\rho_{i, h}^{k}, \rho_{i, h}^{k+1}\right)$. Moreover, $\mathcal{R}$ is defined such that, for all $\phi \in \mathcal{C}_{c}^{\infty}\left([0, T) \times \mathbb{R}^{n}\right)$,

$$
|\mathcal{R}[\phi](x, y)| \leqslant \frac{1}{2}\left\|D^{2} \phi\right\|_{L^{\infty}\left([0, T) \times \mathbb{R}^{n}\right)}|x-y|^{2} .
$$


Combining Propositions 4.1, 4.3, 4.11 and 4.12, the rest of the proof of Theorem 2.3 is identical to the proof of Theorem 2.2 in the previous section.

Remark 4.13. As in Remark 3.8, it is possible to drop one diffusion. Say we drop the individual Entropy for the second species, $\rho_{2}$. The difficulty is to pass to the limit in the nonlinear term $\rho_{2, h} \nabla p_{h}$. This term can be rewritten as

$$
\left(\rho_{1, h}+\rho_{2, h}\right) \nabla p_{h}-\rho_{1, h} \nabla p_{h} .
$$

Taking advantage of the definition of $p_{h}$, we deduce that $\left(\rho_{1, h}+\rho_{2, h}\right) \nabla p_{h}=\nabla p_{h}$ a.e and then converges weakly to $\nabla p=\left(\rho_{1}+\rho_{2}\right) \nabla p$ in $L^{2}((0, T) \times \Omega)$. Moreover, since $\rho_{1, h}$ strongly converges in $L^{2}((0, T) \times \Omega)$ by Proposition 4.3 and $\nabla p_{h}$ converges weakly in $L^{2}((0, T) \times \Omega)$ we can pass to the limit in the second term by strong-weak convergence. Then we deduce that $\rho_{2, h} \nabla p_{h}$ weakly converges to $\rho_{2} \nabla p$.

\section{Systems with a common drift}

In this section, we focus on the special case where $\nabla V_{1}=\nabla V_{2}=: \nabla V \in L^{\infty}(\Omega)$. Although this asumption is very restrictive, it allows us to obtain better estimates on solutions (Proposition 5.2 and Proposition 3.12 which are hard to get in the general case due to the lack of convexity of $\mathcal{F}_{m}\left(\rho_{1}+\rho_{2}\right)$, see Remark 5.1. Therefore, in this case, we will be able to prove the convergence of a solution to (1.3) to a solution to (1.1), when $m$ goes to $+\infty$. Moreover, under some regularity we give a $L^{1}$-contraction result for systems (1.1) and 1.3).

Remark 5.1. It is well-known in the Wasserstein gradient flow theory that the $\lambda$-geodesic convexity of the functional implies a $W_{2}$-contraction of the flow. Unfortunately, as mentioned in [26], in general, $\left(\rho_{1}, \rho_{2}\right) \in \mathcal{P}^{\mathrm{ac}}(\Omega)^{2} \mapsto \mathcal{F}_{m}\left(\rho_{1}+\rho_{2}\right)$ is not displacement convex. Indeed, for $m=2$, we can rewrite the functional as

$$
\mathcal{F}_{2}\left(\rho_{1}+\rho_{2}\right)=\mathcal{F}_{2}\left(\rho_{1}\right)+\mathcal{F}_{2}\left(\rho_{2}\right)+2 \int_{\Omega} \rho_{1} \rho_{2} .
$$

Let $\rho_{2}$ be a fixed density, we study the displacement convexity of $\rho \mapsto \mathcal{F}_{2}(\rho)+2 \int_{\Omega} \rho_{2} \rho$. We know, see [37], that $\rho \in \mathcal{P}^{\mathrm{ac}}(\Omega) \mapsto \mathcal{F}_{2}(\rho)$ is displacement convex but $\rho \mapsto \int_{\Omega} \rho_{2} \rho$ is displacment convex if $\rho_{2}$ is $\lambda$-convex.

To overcome this lack of convexity, we need to obtain a stronger estimate, independent on $m$, on $\nabla F_{m}^{\prime}\left(\rho_{1, m}+\rho_{2, m}\right)$, where $\left(\rho_{1, m}, \rho_{2, m}\right)$ is a solution to 1.3 . In the case of a common drift, this estimate can be found observing that $\rho_{m}:=\rho_{1, m}+\rho_{2, m}$ is the Wasserstein gradient flow of $\mathcal{E}+\mathcal{V}+\mathcal{F}_{m}$ and then, solves

$$
\partial_{t} \mu-\Delta \mu-\operatorname{div}(\mu \nabla V)-\operatorname{div}\left(\mu \nabla F_{m}^{\prime}(\mu)\right)=0,
$$

with initial condition $\mu_{\mid t=0}=\rho_{1,0}+\rho_{2,0}$.

Proposition 5.2. Let $\left(\rho_{1, m}, \rho_{2, m}\right)$ be a solution to (5.1) in $L^{2}\left((0, T), H^{1}(\Omega)\right)$ with $\nabla V_{1}=\nabla V_{2}=$ : $\nabla V \in L^{\infty}(\Omega)$. Then $\rho_{m}:=\rho_{1, m}+\rho_{2, m}$ is unique and $F_{m}^{\prime}\left(\rho_{m}\right)$ is bounded independently of $m$ in $L^{2}\left((0, T), H^{1}(\Omega)\right)$, for all $T<+\infty$.

Proof. As we remark above, $\rho_{m}$ is solution to 5.1). By geodesic convexity of $\mathcal{E}$ and $\mathcal{F}_{m}$, we know that solution to (5.1) is unique (see [3]). To conclude, we reason as in [22, Lemma 5.6]. The proof is based on the flow interchange technique with the (smooth) solution to

$$
\begin{cases}\partial_{t} \eta=\Delta \eta^{m-1}+\varepsilon \Delta \eta & \text { in }(0, T) \times \Omega, \\ \left(\nabla \eta^{m-1}+\varepsilon \nabla \eta\right) \cdot \nu=0 & \text { in }(0, T) \times \partial \Omega, \\ \eta_{\mid t=0}=\rho_{h, m}^{k} & \end{cases}
$$

where $\rho_{h, m}^{k}$ is constructed using the JKO scheme. We obtain, when $\varepsilon$ goes to 0 and using a lower semi-continuity argument, $\left\|\nabla F_{m}^{\prime}\left(\rho_{m}\right)\right\|_{L^{2}\left((0, T), H^{1}(\Omega)\right)} \leqslant C_{T}$, for all $T>0$, where $C_{T}$ is a constant independent on $m$. The $L^{1}$-estimate of $F_{m}^{\prime}\left(\rho_{m}\right)$ and the Poincaré-Wirtinger inequality conclude the proof. 
Now, we show that $\left(\rho_{1, m}, \rho_{2, m}\right)$ converges to a solution to 1.1$),\left(\rho_{1, \infty}, \rho_{2, \infty}\right)$, as $m \nearrow+\infty$.

Theorem 5.3. Assume that the initial data satisfy $\rho_{1,0}+\rho_{2,0} \leqslant 1$. Up to a subsequence, as $m \rightarrow+\infty$, a solution to $[1.3),\left(\rho_{1, m}, \rho_{2, m}\right)$, converges strongly in $L^{2}((0, T) \times \Omega)$ to $\left(\rho_{1, \infty}, \rho_{2, \infty}\right)$ and $p_{m}:=F_{m}^{\prime}\left(\rho_{1, m}+\rho_{2, m}\right)$ converges weakly in $L^{2}\left((0, T), H^{1}(\Omega)\right)$ to $p_{\infty}$, where $\left(\rho_{1, \infty}, \rho_{2, \infty}, p_{\infty}\right)$ is a solution to (1.1).

Proof. First we prove the convergence of $\rho_{i, m}$. We start noticing that the estimate (3.6) does not depend on $m$ and then by Remark 3.2 , we have

$$
\left\|\rho_{i, m}^{1 / 2}\right\|_{L^{2}\left((0, T), H^{1}(\Omega)\right)} \leqslant C_{T} \text { and } W_{2}\left(\rho_{i, m}(t), \rho_{i, m}(s)\right) \leqslant C_{T}|t-s|^{1 / 2},
$$

for all $t, s \leqslant T$ and where $C_{T}$ is a contant independent on $m$. Then using the Rossi-Savaré Theorem we obtain that $\rho_{i, m}$ converges to $\rho_{i, \infty}$ in $L^{1}((0, T) \times \Omega)$. In fact, $\rho_{i, m}$ converges strongly to $\rho_{i, \infty}$ in $L^{2}((0, T) \times \Omega)$. Indeed, for $m \gg 2,\left\|\rho_{i, m}\right\|_{L^{m}((0, T) \times \Omega)}$ is uniformly bounded in $m$ so $\left(\rho_{i, m}^{2}\right)_{m}$ is uniformly integrable. Then, $\rho_{i, m}$ converges weakly in $L^{2}((0, T) \times \Omega)$ to $\rho_{i, \infty}$ and Vitali's convergence Theorem implies that $\left\|\rho_{i, m}\right\|_{L^{2}((0, T) \times \Omega)}=\left\|\rho_{i, m}^{2}\right\|_{L^{1}((0, T) \times \Omega)}^{1 / 2} \rightarrow\left\|\rho_{i, \infty}^{2}\right\|_{L^{1}((0, T) \times \Omega)}^{1 / 2}=$ $\left\|\rho_{i, \infty}\right\|_{L^{2}((0, T) \times \Omega)}$. Furthermore, $p_{m}$ converges weakly in $L^{2}\left((0, T), H^{1}(\Omega)\right)$ to $p_{\infty}$, Proposition 5.2 . and obviously $p_{\infty} \geqslant 0$. Consequently, we can pass to the limit in the weak formulation of the system (1.3) to obtain the weak formulation of sytem (1.1).

To conclude the proof, it remains to prove that

$$
\rho_{1, \infty}+\rho_{2, \infty} \leqslant 1 \text { and } p_{\infty}\left(1-\rho_{1, \infty}-\rho_{2, \infty}\right)=0 \quad \text { a.e. }
$$

We start to show that $\rho_{1, \infty}+\rho_{2, \infty} \leqslant 1$. The argument is the same as in [2, Lemma 4.3]. The estimate 3.3 does not depend on $m$ so we have

$$
\int_{0}^{T} \int_{\Omega}\left(\rho_{1, m}+\rho_{2, m}-1\right)_{+}^{2} d x d t \leqslant \frac{2 C}{m} \rightarrow 0,
$$

when $m \rightarrow+\infty$, which implies that $\rho_{1, \infty}+\rho_{2, \infty} \leqslant 1$ a.e.

To obtain the second part of the claim, we start proving

$$
\int_{0}^{T} \int_{\Omega} p_{m}\left(1-\rho_{1, m}-\rho_{2, m}\right) \varphi d x d t \rightarrow \int_{0}^{T} \int_{\Omega} p_{\infty}\left(1-\rho_{1, \infty}-\rho_{2, \infty}\right) \varphi d x d t,
$$

for all $\varphi \in \mathcal{C}_{c}^{\infty}((0, T) \times \Omega)$. With the same argument as before, $\rho_{1, m}+\rho_{2, m} \rightarrow \rho_{1, \infty}+\rho_{2, \infty}$ strongly in $L^{2}((0, T) \times \Omega)$ and $p_{m} \rightarrow p_{\infty}$ weakly in $L^{2}((0, T) \times \Omega)$, then by strong-weak convergence, we obtain the result. Now, we show that

$$
\int_{0}^{T} \int_{\Omega} p_{m}\left(1-\rho_{1, m}-\rho_{2, m}\right) \varphi d x d t \rightarrow 0,
$$

for all nonnegative $\varphi \in \mathcal{C}_{c}^{\infty}((0, T) \times \Omega)$. We start splitting the integral,

$$
\begin{aligned}
\int_{0}^{T} \int_{\Omega} p_{m}\left(1-\rho_{1, m}-\rho_{2, m}\right) \varphi d x d t & =\iint_{\left\{\rho_{1, m}+\rho_{2, m} \leqslant 1\right\}} p_{m}\left(1-\rho_{1, m}-\rho_{2, m}\right) \varphi d x d t \\
& +\iint_{\left\{\rho_{1, m}+\rho_{2, m} \geqslant 1\right\}} p_{m}\left(1-\rho_{1, m}-\rho_{2, m}\right) \varphi d x d t .
\end{aligned}
$$

Remark that, since $\rho_{1, m}+\rho_{2, m} \rightarrow \rho_{1, \infty}+\rho_{2, \infty}$ strongly in $L^{1}((0, T) \times \Omega)$, up to a subsequence, $\rho_{1, m}(t, x)+\rho_{2, m}(t, x) \rightarrow \rho_{1, \infty}(t, x)+\rho_{2, \infty}(t, x)(t, x)$-a.e. Let $(t, x) \in[0, T] \times \Omega$ be a point where the convergence a.e. holds. If $\rho_{1, \infty}(t, x)+\rho_{2, \infty}(t, x)<1$, then $\rho_{1, m}(t, x)+\rho_{2, m}(t, x) \leqslant(1-\varepsilon)$, for large $m$ and $p_{m}(t, x) \leqslant \frac{m}{m-1}(1-\varepsilon)^{m-1} \rightarrow 0$, therefore $p_{m}(t, x)\left(1-\rho_{1, m}(t, x)-\rho_{2, m}(t, x)\right) \rightarrow 0$. On the other hand, if $\rho_{1, \infty}(t, x)+\rho_{2, \infty}(t, x)=1$ and, for large $m, \rho_{1, m}(t, x)+\rho_{2, m}(t, x) \leqslant 1$, then $1-\rho_{1, m}(t, x)-\rho_{2, m}(t, x) \rightarrow 0$ and $p_{m}(t, x) \leqslant \frac{m}{m-1}$ remains bounded. Thus, $p_{m}(t, x)(1-$ 
$\left.\rho_{1, m}(t, x)-\rho_{2, m}(t, x)\right) \rightarrow 0$ a.e. and since on $\left\{\rho_{1, m}+\rho_{2, m} \leqslant 1\right\}, \rho_{1, m}+\rho_{2, m}$ is bounded by 1 and $p_{m} \leqslant \frac{m}{m-1} \leqslant 2$, by Lebesgue convergence Theorem, we obtain

$$
\iint_{\left\{\rho_{1, m}+\rho_{2, m} \leqslant 1\right\}} p_{m}\left(1-\rho_{1, m}-\rho_{2, m}\right) \varphi d x d t \rightarrow 0 .
$$

The convergence of the second term is obtained by applying Cauchy-Schwarz inequality, (5.2) and Proposition 5.2 .

$$
\left|\iint_{\left\{\rho_{1, m}+\rho_{2, m} \geqslant 1\right\}} p_{m}\left(1-\rho_{1, m}-\rho_{2, m}\right) \varphi d x d t\right| \leqslant\left\|p_{m}\right\|_{L^{2}((0, T) \times \Omega)} \frac{C}{m^{1 / 2}} \rightarrow 0,
$$

when $m \nearrow+\infty$. Then, for all $\varphi \in \mathcal{C}_{c}^{\infty}((0, T) \times \Omega)$,

$$
\int_{0}^{T} \int_{\Omega} p_{\infty}\left(1-\rho_{1, \infty}-\rho_{2, \infty}\right) \varphi d x d t=0 .
$$

Since $p_{\infty}\left(1-\rho_{1, \infty}-\rho_{2, \infty}\right) \geqslant 0$, we conclude that $p_{\infty}\left(1-\rho_{1, \infty}-\rho_{2, \infty}\right)=0$ a.e. in $(0, T) \times \Omega$.

To end this section, we give a $L^{1}$-contraction result for $m \in[1,+\infty]$ under some regularity on solutions but first we establish maximum principle for $m \in[1,+\infty)$.

Proposition 5.4. Assume that $\rho_{i, 0}+\rho_{2,0} \leqslant M_{0}$. For all $m \in[1,+\infty)$ and $T<+\infty$, there exists a constant $M_{T}>0$ such that $\left\|\rho_{1, m}+\rho_{2, m}\right\|_{L^{\infty}((0, T) \times \Omega)} \leqslant M_{T}$. In addition, we have $\nabla \rho_{i, m}, \nabla F_{m}^{\prime}\left(\rho_{1, m}+\rho_{2, m}\right) \in L^{2}((0, T) \times \Omega)$.

Proof. It is well known that the solution $\mu$ to (5.1) satisfies a maximum principle, see for instance [39, 1, 41, 28, 44]. Then by uniqueness of the solution, there exists $M_{T}$ such that $\| \rho_{1, m}+$ $\rho_{2, m} \|_{L^{\infty}((0, T) \times \Omega)} \leqslant M_{T}$. We obtain then

$$
\left|\nabla \rho_{i, m}\right| \leqslant 2 M_{T}^{1 / 2}\left|\nabla \rho_{i, m}^{1 / 2}\right| \text { and }\left|\rho_{i, m} \nabla F_{m}^{\prime}\left(\rho_{1, m}+\rho_{2, m}\right)\right| \leqslant M_{T}\left|\nabla F_{m}^{\prime}\left(\rho_{1, m}+\rho_{2, m}\right)\right| .
$$

Since, $\nabla \rho_{i, m}^{1 / 2}$ and $\nabla F_{m}^{\prime}\left(\rho_{1, m}+\rho_{2, m}\right)$ are in $L^{2}((0, T) \times \Omega)$ (Proposition 5.2 , the proof is concluded.

Remark 5.5. In the sepcial case of a common drift, by Proposition 5.4, we can improve the regularity of solutions to (1.3] in Definition 2.1 if we start with $L^{\infty}$ initial conditions. Then, as in [26. Remark 3.2 (a)], we notice that, by density, we can consider test functions in $W^{1,1}\left((0, T), L^{1}(\Omega)\right) \cap$ $L^{2}\left((0, T), H^{1}(\Omega)\right)$ in Definition 2.1 for system (1.3) and system 1.1.

Theorem 5.6. Let $\left(\rho_{1, m}^{1}, \rho_{2, m}^{1}\right)$ and $\left(\rho_{1, m}^{2}, \rho_{2, m}^{2}\right)$ be two solutions to 1.3 (or 1.1 if $\left.m=+\infty\right)$ with intial conditions $\left(\rho_{1,0}^{1}, \rho_{2,0}^{1}\right)$ and $\left(\rho_{1,0}^{2}, \rho_{2,0}^{2}\right)$, respectively. Assume there exists $M_{0}>0$ such that

$$
\left\|\rho_{1,0}^{1}+\rho_{2,0}^{1}\right\|_{L^{\infty}(\Omega)},\left\|\rho_{1,0}^{2}+\rho_{2,0}^{2}\right\|_{L^{\infty}(\Omega)} \leqslant M_{0} .
$$

If $\partial_{t} \rho_{i, m}^{1}, \partial_{t} \rho_{i, m}^{2} \in L^{1}((0, T) \times \Omega)$, then

$$
\left\|\rho_{i, m}^{1}(t, \cdot)-\rho_{i, m}^{2}(t, \cdot)\right\|_{L^{1}(\Omega)} \leqslant\left\|\rho_{i, 0}^{1}-\rho_{i, 0}^{2}\right\|_{L^{1}(\Omega)} .
$$

Proof. First if $m<+\infty$, since $\rho_{1, m}+\rho_{2, m}$ solves (5.1), then it is unique and according to Proposition $5.2 p_{m}:=F_{m}^{\prime}\left(\rho_{1, m}+\rho_{2, m}\right)$ is in $L^{2}\left((0, T), H^{1}(\Omega)\right)$. Moreover, we have already shown in Theorem 2.3 that the pressure $p_{\infty}$ associated to the constraint $\rho_{1, \infty}+\rho_{2, \infty} \leqslant 1$ is in $L^{2}\left((0, T), H^{1}(\Omega)\right)$ and, according to [21], $\left(\rho_{1, \infty}+\rho_{2, \infty}, p_{\infty}\right)$ is unique. Then, for $m \in[1,+\infty], \rho_{1, m}^{i}$ solves

$$
\partial_{t} \rho_{1, m}^{i}-\Delta \rho_{1, m}^{i}-\operatorname{div}\left(\rho_{1, m}^{i}\left(\nabla V+\nabla p_{m}\right)\right)=0 .
$$

Now, by the same argument as 40, 1, we prove the $L^{1}$-contraction. We prove the result for $i=1$ and the argument is the same for $i=2$. We note $\Omega_{T}:=(0, T) \times \Omega$. Define the smooth function, 
for $z \in \mathbb{R}, f(z)=e^{-1 / z} e^{-1 /(1-z)}$ if $z \in(0,1)$ and 0 otherwise and $M:=\|f\|_{L^{\infty}}$. Then for $\delta>0$, define the smooth function $\phi_{\delta}$ by

$$
\phi_{\delta}(z):=\frac{1}{Z} \int_{0}^{z / \delta} f(\xi) d \xi, \text { where } Z:=\int_{0}^{1} f(\xi) d \xi .
$$

Consider $\zeta_{\delta}:=\phi_{\delta}\left(\rho_{1, m}^{1}-\rho_{1, m}^{2}\right)$. By definition, $\zeta_{\delta} \in W^{1,1}\left((0, T), L^{1}(\Omega)\right) \cap L^{2}\left((0, T), H^{1}(\Omega)\right) \cap$ $L^{\infty}\left(\Omega_{T}\right)$. Then taking $\zeta_{\delta}$ as an admissible test function in Definition 2.1, see Remark 5.5. we obtain

$$
\iint_{\Omega_{T}} \partial_{t}\left(\rho_{1, m}^{1}-\rho_{1, m}^{2}\right) \zeta_{\delta}=-\iint_{\Omega_{T}}\left(\left(\rho_{1, m}^{1}-\rho_{1, m}^{2}\right)\left(\nabla V+\nabla p_{m}\right) \cdot \nabla \zeta_{\delta}+\nabla\left(\rho_{1, m}^{1}-\rho_{1, m}^{2}\right) \cdot \nabla \zeta_{\delta}\right) d x d t .
$$

We introduce $\Omega_{T}^{\delta}:=\Omega_{T} \cap\left\{0<\rho_{1, m}^{1}-\rho_{1, m}^{2}<\delta\right\}$. Then by definition of $\zeta_{\delta}$

$$
\begin{aligned}
& \iint_{\Omega_{T}} \partial_{t}\left(\rho_{1, m}^{1}-\rho_{1, m}^{2}\right) \zeta_{\delta} \\
& \quad=-\frac{1}{Z \delta} \iint_{\Omega_{T}^{\delta}}\left(\rho_{1, m}^{1}-\rho_{1, m}^{2}\right)\left(\nabla V+\nabla p_{m}\right) \cdot \nabla\left(\rho_{1, m}^{1}-\rho_{1, m}^{2}\right) f\left(\frac{\rho_{1, m}^{1}-\rho_{1, m}^{2}}{\delta}\right) d x d t \\
& \quad-\frac{1}{Z \delta} \iint_{\Omega_{T}^{\delta}}\left|\nabla\left(\rho_{1, m}^{1}-\rho_{1, m}^{2}\right)\right|^{2} f\left(\frac{\rho_{1, m}^{1}-\rho_{1, m}^{2}}{\delta}\right) d x d t .
\end{aligned}
$$

Young's inequality gives

$$
\begin{aligned}
& \iint_{\Omega_{T}} \partial_{t}\left(\rho_{1, m}^{1}-\rho_{1, m}^{2}\right) \zeta_{\delta} \\
& \quad \leqslant \frac{M}{2 Z \delta} \iint_{\Omega_{T}^{\delta}}\left(\rho_{1, m}^{1}-\rho_{1, m}^{2}\right)^{2}\left|\nabla V+\nabla p_{m}\right|^{2} d x d t \\
& \quad-\frac{1}{2 Z \delta} \iint_{\Omega_{T}^{\delta}}\left|\nabla\left(\rho_{1, m}^{1}-\rho_{1, m}^{2}\right)\right|^{2} f\left(\frac{\rho_{1, m}^{1}-\rho_{1, m}^{2}}{\delta}\right) d x d t \\
& \quad \leqslant \frac{M}{2 Z}\left\|\nabla V+\nabla p_{m}\right\|_{L^{2}\left(\Omega_{T}\right)}^{2} \delta .
\end{aligned}
$$

Then, when $\delta \searrow 0$, by Fatou's Lemma,

$$
\iint_{\Omega_{T} \cap\left\{\rho_{1, m}^{1}-\rho_{1, m}^{2} \geqslant 0\right\}} \partial_{t}\left(\rho_{1, m}^{1}-\rho_{1, m}^{2}\right) \leqslant 0 .
$$

Reversing the roles of $\rho_{1, m}^{1}$ and $\rho_{1, m}^{2}$, we have

$$
\iint_{\Omega_{T}} \partial_{t}\left(\left|\rho_{1, m}^{1}-\rho_{1, m}^{2}\right|\right) \leqslant 0
$$

which concludes the proof.

\section{$6 \quad$ Numerical simulations}

To end this paper, we use the algorithm introduced in [6] to present numerical simulations in dimension 2 on the square $\Omega=\left[-\frac{1}{2}, \frac{1}{2}\right]^{2}$. Simulations are carried out using a $50 \times 50$ discretization in space with a time step $h=0.01$. The first system we study is the transport equation with common porous media congestion, without individual diffusions,

$$
\partial_{t} \rho_{i}-\alpha_{i} \operatorname{div}\left(\rho_{i} \nabla F_{m}^{\prime}\left(\alpha_{1} \rho_{1}+\alpha_{2} \rho_{2}\right)\right)-\operatorname{div}\left(\rho_{i} \nabla V_{i}\right)=0, i=1,2,
$$


which, at least formally, is the gradient flow in Wasserstein space for the energy

$$
\mathcal{E}\left(\rho_{1}, \rho_{2}\right):=\int_{\Omega} V_{1} \rho_{1}+\int_{\Omega} V_{2} \rho_{2}+\int_{\Omega} F_{m}\left(\alpha_{1} \rho_{1}+\alpha_{2} \rho_{2}\right) .
$$

Arguing as in [6], setting $\phi=\left(\phi_{1}, \phi_{2}\right),\left(D \phi_{1}, D \phi_{2}\right):=\left(\partial_{t} \phi_{1}, \nabla \phi_{1}, \partial_{t} \phi_{2}, \nabla \phi_{2}\right), q=\left(q_{1}, q_{2}\right)=$ $\left(a_{1}, b_{1}, c_{1}, a_{2}, b_{2}, c_{2}\right), \sigma=\left(\sigma_{1}, \sigma_{2}\right)=\left(\left(\mu_{1}, m_{1}, \widetilde{\mu}_{1}\right),\left(\mu_{2}, m_{2}, \widetilde{\mu}_{2}\right)\right)$ and defining the convex set $K:=$ $\left\{(a, b) \in \mathbb{R}^{n+1}: a+\frac{1}{2}|b|^{2} \leqslant 0\right\}$, one can rewrite one step of the JKO scheme, 3.1), with $\mathcal{E}$ as a saddle-point problem for the augmented Lagrangian

$$
\begin{aligned}
L_{r}(\phi, q, \sigma) & =\sum_{i=1}^{2} \int_{\Omega} \phi_{i}(0, x) \rho_{i, h}^{k}(x) \mathrm{d} x+\sum_{i=1}^{2} \int_{0}^{1} \int_{\Omega} \chi_{K}\left(a_{i}(t, x), b_{i}(t, x)\right) \mathrm{d} x \mathrm{~d} t \\
& +\sum_{i=1}^{2} \int_{0}^{1} \int_{\Omega}\left(\left(\mu_{i}, m_{i}\right) \cdot\left(D \phi_{i}-\left(a_{i}, b_{i}\right)\right)+\frac{r}{2}\left|D \phi_{i}-\left(a_{i}, b_{i}\right)\right|^{2}\right) \mathrm{d} x \mathrm{~d} t \\
& +\sum_{i=1}^{2} \int_{\Omega}\left(\frac{r}{2}\left|\phi_{i}(1, x)+c_{i}(x)\right|^{2} \mathrm{~d} x-\left(\phi_{i}(1, x)+c_{i}(x)\right) \widetilde{\mu}_{i}(x)\right) \mathrm{d} x \\
& +h \mathcal{E}^{*}\left(\frac{c_{1}}{h}, \frac{c_{2}}{h}\right),
\end{aligned}
$$

where $\mathcal{E}^{*}$ is the Legendre tranform of $\mathcal{E}$ extended by $+\infty$ on $(-\infty, 0]$. A saddle point of $L_{r}$ satisfies $\mu_{i}(1, \cdot)=\tilde{\mu}_{i}$ and the solution to one JKO step is $\rho_{i, h}^{k+1}=\tilde{\mu}_{i}$. Then, we use the augmented Lagrangian algorithm, ALG2-JKO, introduced in [6] to compute numerically $\left(\rho_{1, h}^{k+1}, \rho_{2, h}^{k+1}\right)$ and we refer to [6] for a detailed exposition.

Figure 11 represents two populations crossing each other subject to porous media congestion with $\alpha_{1}=\alpha_{2}=1$ and $m=50$. Initial conditions are given by

$$
\rho_{1,0}=\mathbb{1}_{[-0.45,-0.15]^{2}} \text { and } \rho_{2,0}=\mathbb{1}_{[0.15,0.45]^{2}} .
$$

The motion is imposed by potentials $V_{1}(x, y)=4\|(x, y)-(0.3,0.3)\|^{2}$ and $V_{2}(x, y)=4 \|(x, y)+$ $(0.3,0.3) \|^{2}$. We remark that the two populations have the same behaviour and when they cross each other, the density has to spread. In Figure2, we study the same behaviour but subject to the porous medium constraint on $\rho_{1}+2 \rho_{2}$. We can see that the population where the constraint plays a higher role, $\rho_{2}$, has to deviate in order to let pass $\rho_{1}$ through. Although the theory is not fully understood for system (6.1) (see discusions in [26]), we notice that in Figures 1 and 2, it seems that the unique discrete solutions behave numerically stable.

In the two populations crowd motion model with linear diffusion, we saw that we can find a solution as the gradient flow of

$$
\mathcal{E}\left(\rho_{1}, \rho_{2}\right):=\int_{\Omega}\left(V_{1}+\varepsilon \log \left(\rho_{1}\right)\right) \rho_{1}+\int_{\Omega}\left(V_{2}+\varepsilon \log \left(\rho_{2}\right)\right) \rho_{2}+\mathcal{F}_{\infty}\left(\alpha_{1} \rho_{1}+\alpha_{2} \rho_{2}\right) .
$$

In this context, we use the same initial datas and potentials as previously. The small parameter $\varepsilon=0.01$ in the simulations is taken to reduce the effect of the diffusion. In Figure 3 we see two populations which cross each other. When they start to cross each other at time $t=0.05$, we remark that the density of $\rho_{1}$ and $\rho_{2}$ decrease and the sum is saturated. In this situation, individuals of both populations take the same space.

Now assume that an individual of the second population takes twice the space than an individual of the first population. Then if we study the one population model (without interaction), populations $\rho_{1}$ and $\rho_{2}$ are subject to constraints $\rho_{1}(x) \leqslant 1$ and $\rho_{2}(x) \leqslant \frac{1}{2}$. In our case, where populations interact each other, $\rho_{1}$ and $\rho_{2}$ are subject to the common constraint $\rho_{1}(x)+2 \rho_{2}(x) \leqslant 1$. Notice that when $\rho_{1}(x)=0$ or $\rho_{2}(x)=0$, we recover the expected behaviour, $\rho_{2}(x) \leqslant \frac{1}{2}$ and $\rho_{1}(x) \leqslant 1$. In Figure 4 , we represent two populations crossing each other subject to this constraint. Immediately, the second population sprawls to saturate the constraints $\rho_{2}(x) \leqslant \frac{1}{2}$ and then when they start crossing the density of $\rho_{1}$ and $\rho_{2}$ decrease and we have $\rho_{1}(x)+2 \rho_{2}(x)=1$.

In Figures 5 and 6 , the same situations as in Figures 3 and 4 are presented adding an obstacle in the middle of $\Omega$. This can be done using a potential with very high value in this area. 

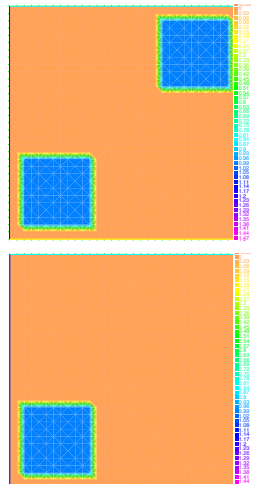

$t=0$
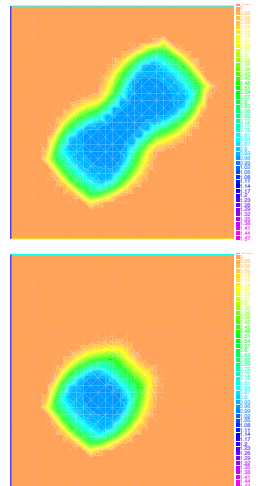

$t=0.05$
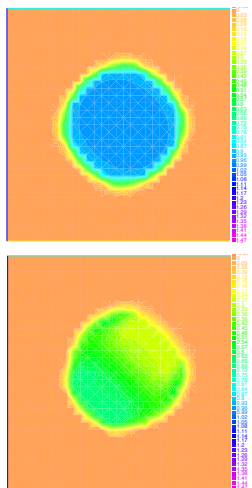

$t=0.1$
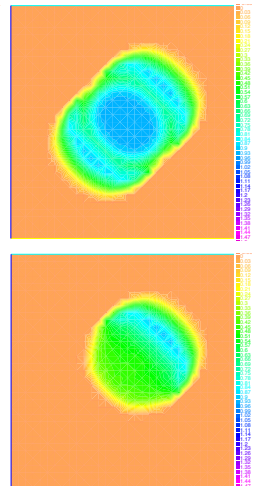

$t=0.15$
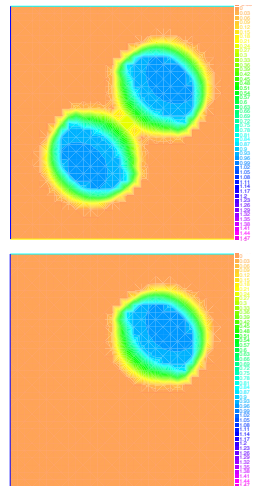

$t=0.2$
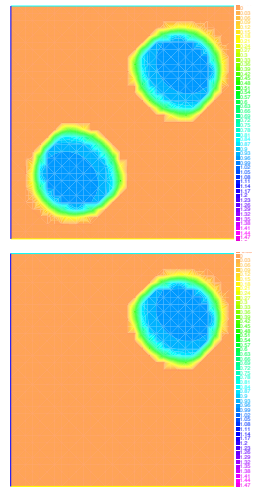

$t=0.3$

Figure 1: Evolution of two species crossing each other with porous media congestion, $m=50$. Top row: display of $\rho_{1}+\rho_{2}$. Bottom row: display of $\rho_{1}$.
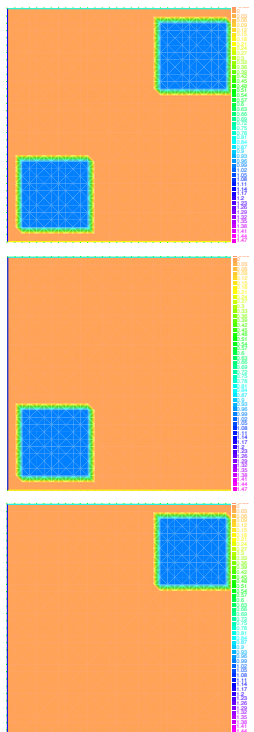

$t=0$
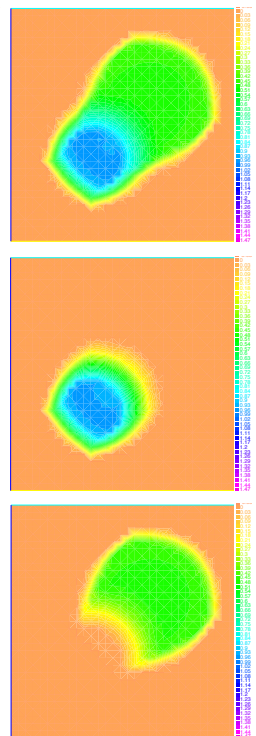

$t=0.05$
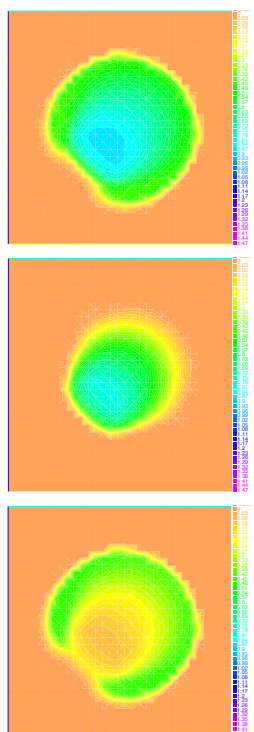

$t=0.1$
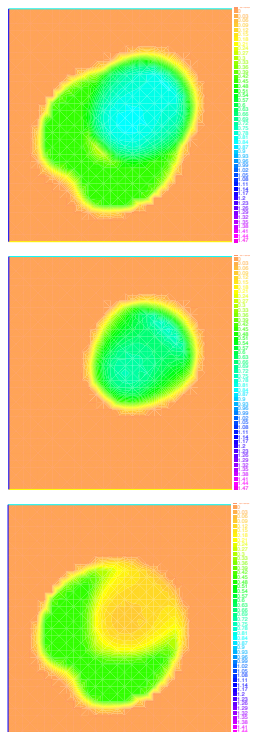

$t=0.15$
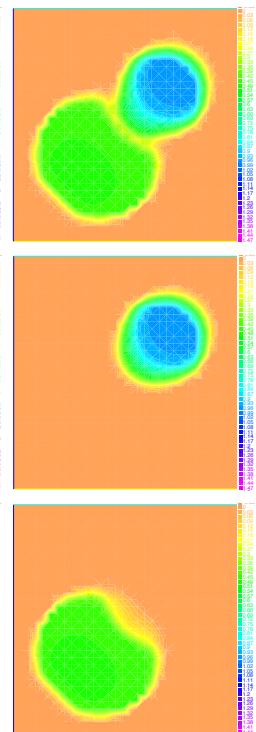

$t=0.2$
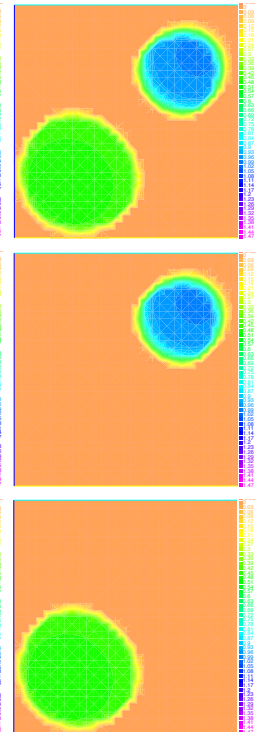

$t=0.3$

Figure 2: Evolution of two species crossing each other with weighted porous media congestion, $\left(\rho_{1}+2 \rho_{2}\right)^{m}, m=50$. Top row: display of $\rho_{1}+\rho_{2}$. Middle row: display of $\rho_{1}$. Bottom row: display of $\rho_{2}$.

\section{Acknowledgements}

The author gratefully thanks G. Carlier for suggesting this problem and for fruitful discussions about this work.

\section{References}

[1] M. Agueh. Existence of solutions to degenerate parabolic equations via the Monge-Kantorovich theory. Adv. Differential Equations, 10(3):309-360, 2005.

[2] D. Alexander, I. Kim, and Y. Yao. Quasi-static evolution and congested crowd transport. Nonlinearity, 27(4):823-858, 2014.

[3] L. Ambrosio, N. Gigli, and G. Savaré. Gradient flows in metric spaces and in the space of probability measures. Lectures in Mathematics ETH Zürich. Birkhäuser Verlag, Basel, 2005. 

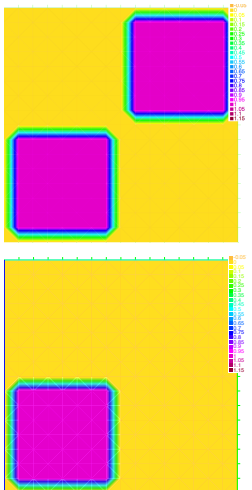

$t=0$
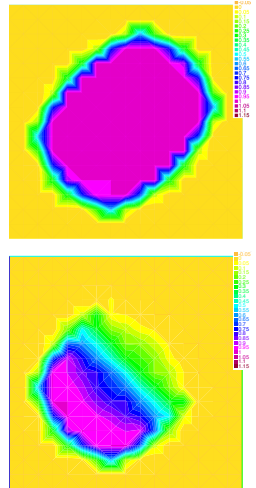

$t=0.05$
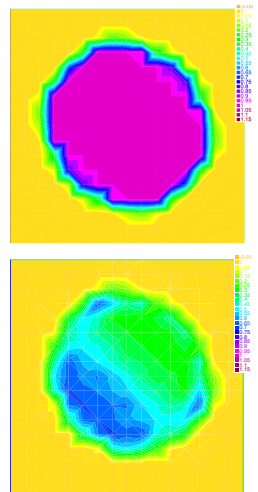

$t=0.1$
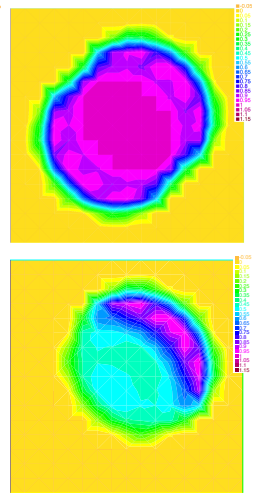

$t=0.15$
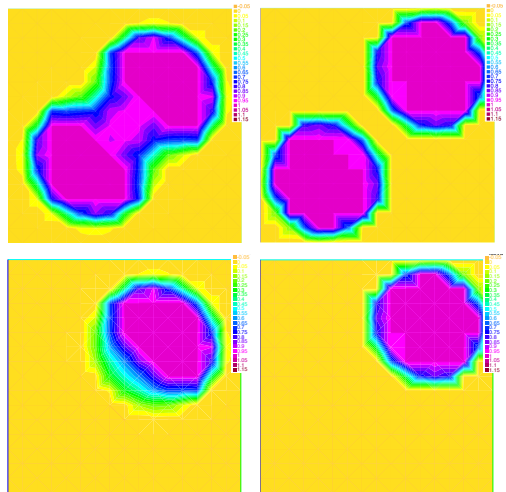

$t=0.2$

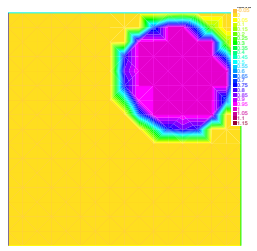

$t=0.3$

Figure 3: Evolution of two species crossing each other with density constraint. Top row: display of $\rho_{1}+\rho_{2}$. Bottom row: display of $\rho_{1}$.
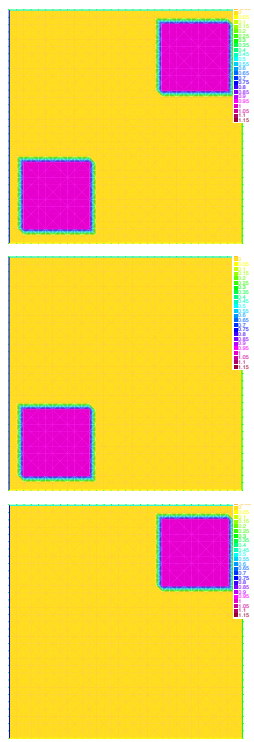

$t=0$
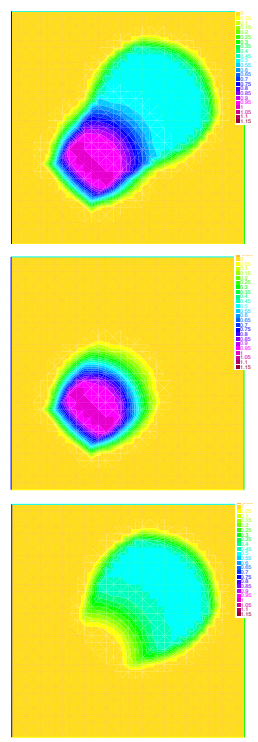

$t=0.05$
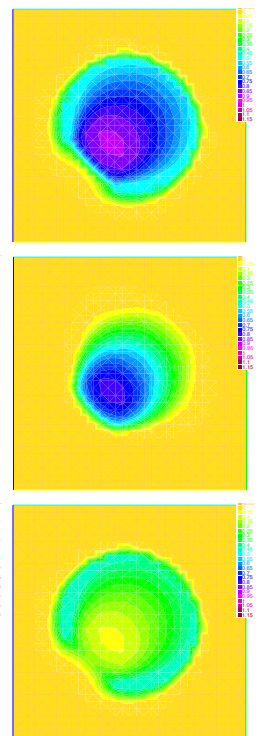

$t=0.1$
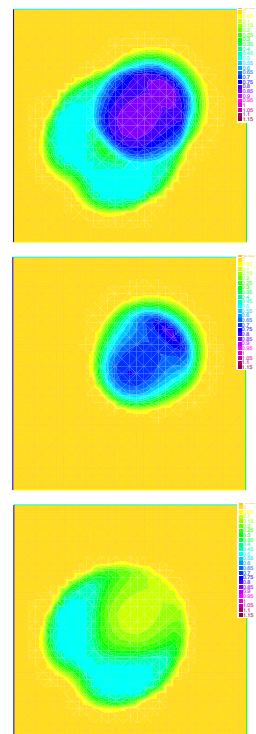

$t=0.15$
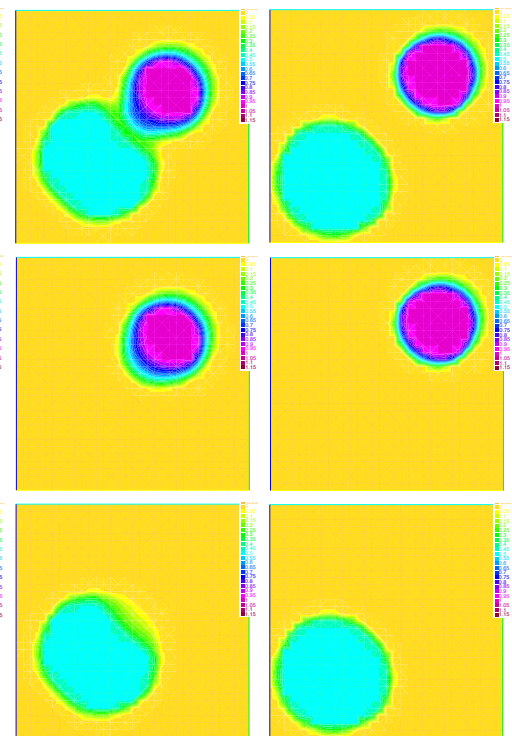

$t=0.2$

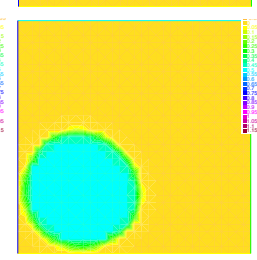

$t=0.3$

Figure 4: Evolution of two species crossing each other with weighted density constraint, $\rho_{1}+2 \rho_{2} \leqslant 1$. Top row: display of $\rho_{1}+\rho_{2}$. Middle row: display of $\rho_{1}$. Bottom row: display of $\rho_{2}$.

[4] A. Bakhta and V. Ehrlacher. Cross-diffusion systems with non-zero flux and moving boundary conditions. November 2016. preprint.

[5] J.-D. Benamou, Y. Brenier, and K. Guittet. Numerical analysis of a multi-phasic mass transport problem. Contemporary Mathematics, 353:1-18, 2004.

[6] J.-D. Benamou, G. Carlier, and M. Laborde. An augmented Lagrangian approach to Wasserstein gradient flows and applications. In Gradient flows: from theory to application, volume 54 of ESAIM Proc. Surveys, pages 1-17. EDP Sci., Les Ulis, 2016.

[7] Y. Brenier. Polar factorization and monotone rearrangement of vector-valued functions. Comm. Pure Appl. Math., 44(4):375-417, 1991.

[8] G. Buttazzo and F. Santambrogio. A model for the optimal planning of an urban area. SIAM J. Math. Anal., 37(2):514-530, 2005.

[9] C. Cancès, T. O. Gallouët, and L. Monsaingeon. Incompressible immiscible multiphase flows in porous media: a variational approach. Anal. PDE, 10(8):1845-1876, 2017. 

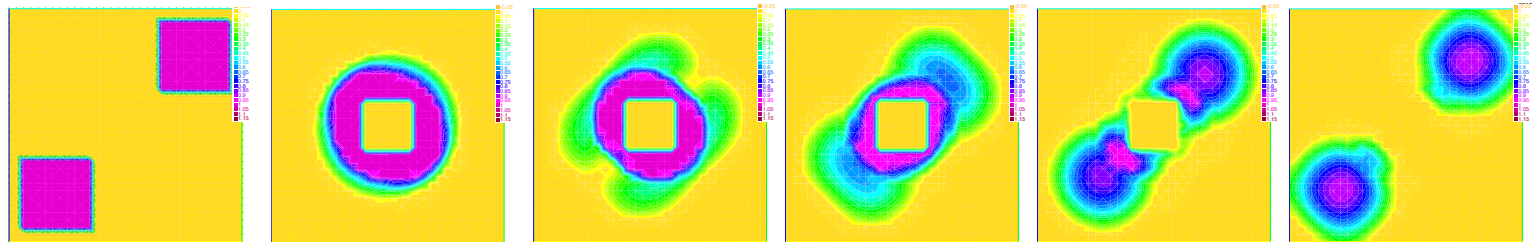

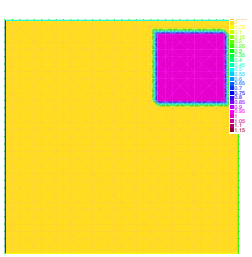

$t=0$

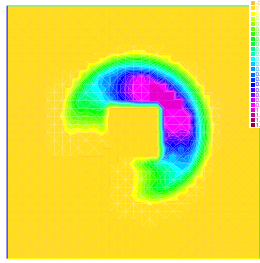

$t=0.1$

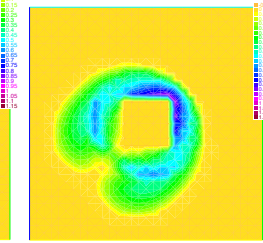

$t=0.2$

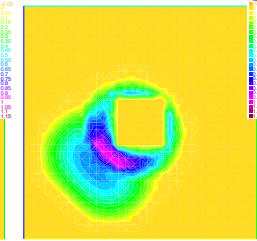

$t=0.3$

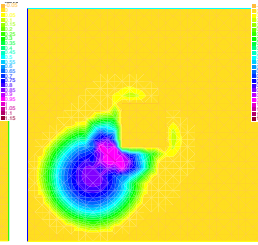

$t=0.4$

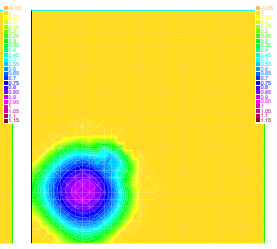

$t=0.5$

Figure 5: Evolution of two species crossing each other with density constraint and an obstacle. Top row: display of $\rho_{1}+\rho_{2}$. Bottom row: display of $\rho_{1}$.
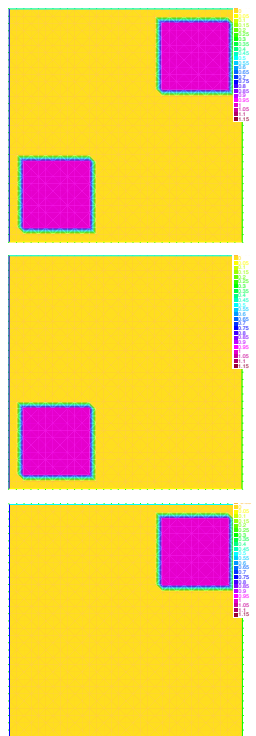

$t=0$
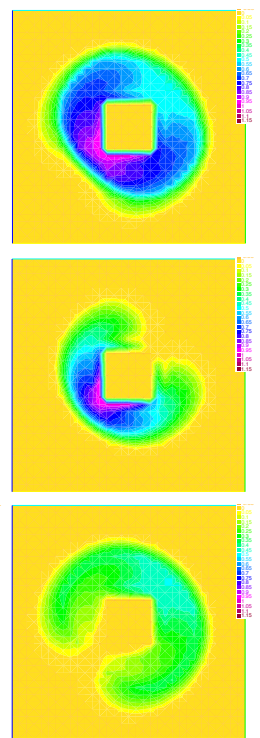

$t=0.1$
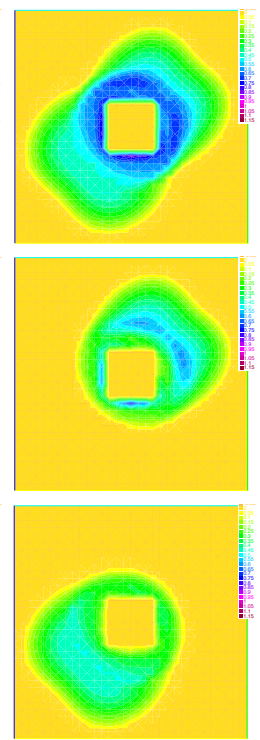

$t=0.2$
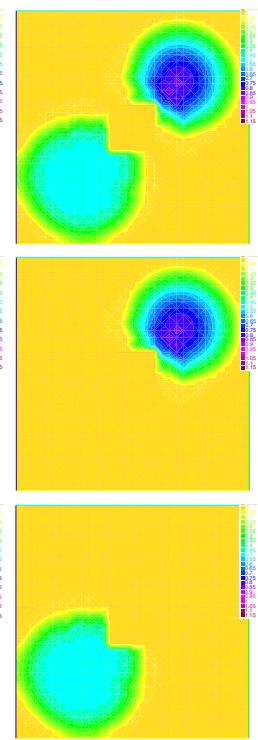

$t=0.3$
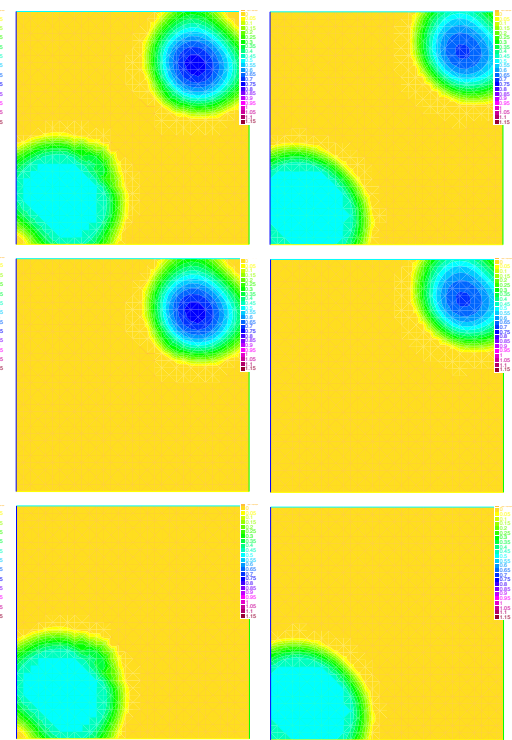

$t=0.4$

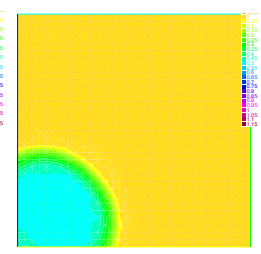

$t=0.5$

Figure 6: Evolution of two species crossing each other with weighted density constraint, $\rho_{1}+2 \rho_{2} \leqslant 1$, and an obstacle. Top row: display of $\rho_{1}+\rho_{2}$. Middle row: display of $\rho_{1}$. Bottom row: display of $\rho_{2}$.

[10] G. Carlier and M. Laborde. A splitting method for nonlinear diffusions with nonlocal, nonpotential drifts. Nonlinear Analysis: Theory, Methods $\&$ Applications, 150:1 - 18, 2017.

[11] G. Carlier and F. Santambrogio. A variational model for urban planning with traffic congestion. ESAIM Control Optim. Calc. Var., 11(4):595-613, 2005.

[12] R. M. Colombo, M. Garavello, and M. Lécureux-Mercier. A class of nonlocal models for pedestrian traffic. Math. Models Methods Appl. Sci., 22(4):1150023, 34, 2012.

[13] R. M. Colombo and M. Lécureux-Mercier. Nonlocal crowd dynamics models for several populations. Acta Math. Sci. Ser. B Engl. Ed., 32(1):177-196, 2012.

[14] G. Crippa and M. Lécureux-Mercier. Existence and uniqueness of measure solutions for a system of continuity equations with non-local flow. NoDEA Nonlinear Differential Equations Appl., 20(3):523-537, 2013. 
[15] J. Dambrine, N. Meunier, B. Maury, and A. Roudneff-Chupin. A congestion model for cell migration. Commun. Pure Appl. Anal., 11(1):243-260, 2012.

[16] S. Daneri and G. Savaré. Eulerian calculus for the displacement convexity in the Wasserstein distance. SIAM J. Math. Anal., 40(3):1104-1122, 2008.

[17] D. G. De Figueiredo. Lectures on the Ekeland variational principle with applications and detours. Springer Berlin, 1989.

[18] L. Desvillettes, T. Lepoutre, A. Moussa, and A. Trescases. On the entropic structure of reaction-cross diffusion systems. Comm. Partial Differential Equations, 40(9):1705-1747, 2015.

[19] M. Di Francesco and S. Fagioli. Measure solutions for non-local interaction PDEs with two species. Nonlinearity, 26(10):2777-2808, 2013.

[20] M. Di Francesco and D. Matthes. Curves of steepest descent are entropy solutions for a class of degenerate convection-diffusion equations. Calc. Var. Partial Differential Equations, 50(1-2):199-230, 2014.

[21] S. Di Marino and A. R. Mészáros. Uniqueness issues for evolution equations with density constraints. Math. Models Methods Appl. Sci., 26(9):1761-1783, 2016.

[22] T. O. Gallouët, M. Laborde, and L. Monsaingeon. An unbalanced optimal transport splitting scheme for general advection-reaction-diffusion problems. to appear in ESAIM Control. Optim. Calc. Var., 2017.

[23] R. Jordan, D. Kinderlehrer, and F. Otto. The variational formulation of the Fokker-Planck equation. SIAM J. Math. Anal., 29(1):1-17, 1998.

[24] A. Jüngel. The boundedness-by-entropy method for cross-diffusion systems. Nonlinearity, 28(6):1963-2001, 2015.

[25] A. Jüngel and N. Zamponi. A cross-diffusion system derived from a Fokker-Planck equation with partial averaging. Z. Angew. Math. Phys., 68(1):Art. 28, 15, 2017.

[26] I. Kim and A. R. Mészáros. On nonlinear cross-diffusion systems: an optimal transport approach. Calc. Var. Partial Differential Equations, 57(3):79, 2018.

[27] S. Kondratyev, L. Monsaingeon, and D. Vorotnikov. A fitness-driven cross-diffusion system from population dynamics as a gradient flow. J. Differential Equations, 261(5):2784-2808, 2016.

[28] M. Laborde. Interacting particles systems, Wasserstein gradient flow approach. PhD thesis, Paris-Dauphine University, 2016.

[29] M. Laborde. On some nonlinear evolution systems which are perturbations of Wasserstein gradient flows. In Topological optimization and optimal transport, volume 17 of Radon Ser. Comput. Appl. Math., pages 304-332. De Gruyter, Berlin, 2017.

[30] P. Laurençot and B.-V. Matioc. A gradient flow approach to a thin film approximation of the Muskat problem. Calc. Var. Partial Differential Equations, 47(1-2):319-341, 2013.

[31] T. Lepoutre, M. Pierre, and G. Rolland. Global well-posedness of a conservative relaxed cross diffusion system. SIAM J. Math. Anal., 44(3):1674-1693, 2012.

[32] D. Matthes, R. J. McCann, and G. Savaré. A family of nonlinear fourth order equations of gradient flow type. Comm. Partial Differential Equations, 34(10-12):1352-1397, 2009.

[33] B. Maury, A. Roudneff-Chupin, and F. Santambrogio. A macroscopic crowd motion model of gradient flow type. Math. Models Methods Appl. Sci., 20(10):1787-1821, 2010.

[34] B. Maury, A. Roudneff-Chupin, and F. Santambrogio. Congestion-driven dendritic growth. Discrete Contin. Dyn. Syst., 34(4):1575-1604, 2014. 
[35] B. Maury, A. Roudneff-Chupin, F. Santambrogio, and J. Venel. Handling congestion in crowd motion modeling. Netw. Heterog. Media, 6(3):485-519, 2011.

[36] B. Maury and J. Venel. Handling of Contacts in Crowd Motion Simulations, pages 171-180. Springer Berlin Heidelberg, 2009.

[37] R. J. McCann. A convexity principle for interacting gases. Adv. Math., 128(1):153-179, 1997.

[38] A. R. Mészáros and F. Santambrogio. Advection-diffusion equations with density constraints. Anal. PDE, 9(3):615-644, 2016.

[39] F. Otto. Double Degenerate Diffusion Equations as Steepest Descent. Preprint. Bonn University, 1996.

[40] F. Otto. $L^{1}$-contraction and uniqueness for quasilinear elliptic-parabolic equations. J. Differential Equations, 131(1):20-38, 1996.

[41] L. Petrelli and A. Tudorascu. Variational principle for general diffusion problems. Appl. Math. Optim., 50(3):229-257, 2004.

[42] R. Rossi and G. Savaré. Tightness, integral equicontinuity and compactness for evolution problems in Banach spaces. Ann. Sc. Norm. Super. Pisa Cl. Sci. (5), 2(2):395-431, 2003.

[43] F. Santambrogio. Gradient flows in Wasserstein spaces and applications to crowd movement. In Seminaire: Equations aux Dérivées Partielles. 2009-2010, Sémin. Équ. Dériv. Partielles, pages Exp. No. XXVII, 16. École Polytech., Palaiseau, 2012.

[44] F. Santambrogio. Optimal Transport for Applied Mathematicians. Progress in Nonlinear Differential Equations and Their Applications 87. Birkasauser Verlag, Basel, 2015.

[45] C. Villani. Topics in optimal transportation, volume 58 of Graduate Studies in Mathematics. American Mathematical Society, Providence, RI, 2003.

[46] C. Villani. Optimal transport, volume 338 of Grundlehren der Mathematischen Wissenschaften [Fundamental Principles of Mathematical Sciences]. Springer-Verlag, Berlin, 2009. Old and new. 